\title{
A HÖLDER INFINITY LAPLACIAN
}

\author{
Antonin Chambolle ${ }^{1}$, Erik Lindgren $^{2}$ and Régis Monneau $^{3}$
}

\begin{abstract}
In this paper we study the limit as $p \rightarrow \infty$ of minimizers of the fractional $W^{s, p}$-norms. In particular, we prove that the limit satisfies a non-local and non-linear equation. We also prove the existence and uniqueness of solutions of the equation. Furthermore, we prove the existence of solutions in general for the corresponding inhomogeneous equation. By making strong use of the barriers in this construction, we obtain some regularity results.
\end{abstract}

Mathematics Subject Classification. 35D40, 35J60, 35J65.

Received April 7, 2011.

\section{INTRODUCTION AND MAIN RESULT}

\subsection{Setting of the problem}

Let $\Omega$ be a bounded open set in $\mathbb{R}^{N}$. Under suitable conditions, it is well-known that if $u_{p}$ minimizes the integral

$$
\int_{\Omega}|\nabla u|^{p}
$$

then $u_{p} \rightarrow u$ as $p \rightarrow \infty$ where $u$ solves the equation

$$
\Delta_{\infty} u=\sum_{i, j=1, \ldots, N} u_{i j} u_{i} u_{j}=0 \quad \text { on } \quad \Omega
$$

with $u_{i}=\frac{\partial u}{\partial x_{i}}$ and $u_{i j}=\frac{\partial^{2} u}{\partial x_{i} \partial x_{j}}$, which is usually referred to as the infinity Laplace equation. See for instance [1,5] for discussions concerning this passage to the limit. Moreover, $u$ is known to be a local minimizer of the Lipschitz norm, i.e., a Lipschitz extension. A lot of the known results concerning infinity harmonic functions and Lipschitz extensions can be found in [3]. Some explicit Lipschitz extensions can be found in [13,17], and these are in general not infinity harmonic functions. Lipschitz extensions have been given a lot of attention recently, and as possible applications one has suggested for instance image interpolation (cf. [8]) and brain warping (cf. [14]).

Keywords and phrases. Lipschitz extensions, Hölder extensions, infinity Laplacian, non-local and non-linear equations, viscosity solutions.

1 CMAP, École Polytechnique, 91128 Palaiseau Cedex, France. antonin.chambolle@cmap.polytechnique.fr

2 Dept. of Mathematical Sciences, NTNU, 7491 Trondheim, Norway. erik.lindgren@math.ntnu.no

3 Université Paris-Est, Cermics, École des Ponts ParisTech, 6-8, avenue Blaise-Pascal, 77455 Marne-la-Vallée Cedex 2, France. monneau@cermics.enpc.fr 
In the present paper, we address the following question:

What happens if we replace the space $W^{1, p}(\Omega)$ by $W^{s, p}(\Omega)$ with $s \in(0,1)$ ?

We study minimizers of the functional

$$
\int_{\Omega \times \Omega} \frac{|u(x)-u(y)|^{p}}{|x-y|^{\alpha p}} \mathrm{~d} x \mathrm{~d} y
$$

for $\alpha \in(0,1]$. We see that this is the $W^{s, p}$-norm for $s=\alpha-N / p$, and the form of the functional suggests that in the limit we should obtain a local minimizer of the $\alpha$-Hölder semi-norm. The Euler-Lagrange equation of this functional is

$$
\int_{\Omega}\left|\frac{u(x)-u(y)}{|x-y|^{\alpha}}\right|^{p-1} \frac{\operatorname{sgn}(u(x)-u(y))}{|x-y|^{\alpha}} \mathrm{d} y=0 .
$$

Formally, one can see that, as $p \rightarrow \infty$, this should converge to the equation

$$
L u=0 \text { in } \Omega
$$

with the operator

$$
(L u)(x)=\sup _{y \in \bar{\Omega}, y \neq x} \frac{u(y)-u(x)}{|y-x|^{\alpha}}+\inf _{y \in \bar{\Omega}, y \neq x} \frac{u(y)-u(x)}{|y-x|^{\alpha}} \text { for } \quad x \in \Omega
$$

that we call the Hölder infinity laplacian. In this paper, we study the Dirichlet problem

$$
\left\{\begin{array}{l}
L u=f \text { in } \Omega \\
u=g \text { on } \partial \Omega .
\end{array}\right.
$$

We obtain existence and some regularity results for this problem in general. In the case $f=0$, we are also able to obtain uniqueness and an implicit representation formula of the solution. Moreover, we prove that the solution is an optimal Hölder extension, in the sense that the Hölder seminorm in $\Omega$ is always less than or equal to the one for the boundary data given on $\partial \Omega$.

At a first glance one might believe that for $\alpha=1$, the Hölder infinity Laplace equation is equivalent to the infinity Laplace equation. This is not the case in general. Indeed, using (ii) in Theorem 1.5 one can quite easily see that the infinity harmonic function

$$
u(x)=\left|x_{1}\right|^{\frac{4}{3}}-\left|x_{2}\right|^{\frac{4}{3}},
$$

found by Aronsson (cf. [2]), is not a solution of (1.4) for $\alpha=1$ and

$$
\Omega=\{-2 \leq x \leq 2,-1 \leq y \leq 1\}
$$

Many of these results are also valid in the case when we replace $\Omega$ by $\mathbb{R}^{n}$ in the sense that we consider minimizers of

$$
\int_{\mathbb{R}^{n} \times \mathbb{R}^{n}} \frac{|u(x)-u(y)|^{p}}{|x-y|^{\alpha p}} \mathrm{~d} x \mathrm{~d} y
$$

with prescribed values $u=g$ in $\mathbb{R}^{n} \backslash \Omega$ and under some appropriate growth condition on $g$ at infinity. Then the limiting operator will instead be

$$
\sup _{y \in \mathbb{R}^{n}, y \neq x} \frac{u(y)-u(x)}{|y-x|^{\alpha}}+\inf _{y \in \mathbb{R}^{n}, y \neq x} \frac{u(y)-u(x)}{|y-x|^{\alpha}} \text { for } x \in \Omega .
$$


If $\Omega \neq \mathbb{R}^{n}$ this operator does not coincide with the infinity Laplace operator. Indeed, the operator above will change if we change the values of $g$ away from $\Omega$, which is not the case for the infinity Laplace operator. Very recently, in [6], a closely related operator has been studied. There the authors consider a non-local "tug-ofwar" game, which in the limit yields an operator also producing optimal Hölder extensions. Moreover, when a parameter is chosen correctly, this operator coincides with the infinity Laplace operator.

\subsection{Main results}

In all that follows, for $\alpha \in(0,1]$, we will denote the $\alpha$-Hölder semi-norm of a function $f$ defined on $A \subset \mathbb{R}^{N}$ by

We also recall the notation

$$
[f]_{\alpha, \Omega}=\sup _{x, y \in A, x \neq y} \frac{|f(x)-f(y)|}{|x-y|^{\alpha}}
$$

$$
C^{0, \alpha}(A)=\left\{f \in C(A),\|f\|_{L^{\infty}(A)}+[f]_{\alpha, A}<\infty\right\},
$$

where $C(A)$ is the set of continuous function on $A$.

The first main result in this paper states that what we expect actually happens when we pass to the limit $p \rightarrow \infty$, as long as the integrals make sense.

Theorem 1.1 (limit equation as $p \rightarrow \infty)$. Let $\alpha \in(0,1]$ and if $\alpha=1$ assume $N \geq 2$. Consider a bounded Lipschitz domain $\Omega$ in $\mathbb{R}^{N}$, and boundary data $g \in C^{0, \alpha}(\partial \Omega)$. For any $p>2 N / \alpha$, there exists a unique minimizer $u_{p}$ of (1.1) satisfying $u=g$ on $\partial \Omega$. Moreover, as $p \rightarrow \infty$, we have $u_{p} \rightarrow u_{\infty}$ uniformly in $\bar{\Omega}$ and $u_{\infty} \in C^{0, \alpha}(\bar{\Omega})$ is a viscosity solution of (1.3).

Remark 1.2. The reason why we haven't treated the case $\alpha=N=1$ is simply that the Euler-Lagrange equation (1.2) is not well defined in a pointwise sense in this case.

Remark 1.3. If $\alpha=\alpha_{p} \rightarrow \alpha_{\infty}<1$, the proof can easily be adapted to obtain a result similar to Theorem 1.1.

Remark 1.4. The reader might wonder why the assumption that $\Omega$ is a Lipschitz domain is necessary. The reason is that we at some point need to apply a fractional version of the Sobolev embedding, which, to the authors knowledge, is known only in the case when $\Omega$ is a bounded Lipschitz domain.

More generally we can consider the inhomogeneous Dirichlet problem

$$
\left\{\begin{array}{l}
L u=f \text { in } \Omega \\
u=g \text { on } \partial \Omega
\end{array}\right.
$$

for which the notion of viscosity solutions is given in Definition 4.1. Then, when $f=0$, there exists a representation formula for $u$.

Theorem 1.5 (existence for general $f$, partial uniqueness). Let $\alpha \in(0,1], \Omega$ be a bounded open set, $g \in C(\partial \Omega)$ and $f \in C(\Omega) \cap L^{\infty}(\Omega)$.

(i) (Existence) Then there exists a viscosity solution $u \in C(\bar{\Omega})$ of $(1.5)$.

(ii) (Partial uniqueness) Assume $f=0$. Then the viscosity solution $u \in C(\bar{\Omega})$ of $(1.5)$ is unique and is defined implicitly by the following:

$$
u(x)=\left\{\begin{array}{ll}
g(x) & \text { if } \quad x \in \partial \Omega \\
a \text { with } \quad \ell_{x}(a)=0 & \text { if } \quad x \in \Omega
\end{array},\right.
$$

where

$$
\ell_{x}(a)=\sup _{y \in \partial \Omega} \frac{g(y)-a}{|y-x|^{\alpha}}+\inf _{y \in \partial \Omega} \frac{g(y)-a}{|y-x|^{\alpha}} .
$$


Remark 1.6. The solution defined by (1.6) is the same as the Lipschitz extension introduced by Oberman in [16] for the distance $d(x, y)=|x-y|^{\alpha}$.

Remark 1.7. It is not clear whether the uniqueness holds for general functions $f$ or not. For the inhomogeneous infinity Laplace equation, the uniqueness is only known to hold if $f$ does not change sign, see [12], where also a counterexample to the uniqueness for $f$ changing sign is provided.

Finally we are also able to obtain the following regularity results, where we use the notation

$$
\operatorname{diam} \Omega=\sup \{|x-y|, x, y \in \Omega\} .
$$

Theorem 1.8 (regularity). Let $\alpha \in(0,1], \Omega$ be a bounded open set, $g \in C(\partial \Omega), f \in C(\Omega) \cap L^{\infty}(\Omega)$ and $u \in C(\bar{\Omega})$ a viscosity solution of (1.5).

(i) For any $K \subset \subset \Omega$ and any $0<\beta<\alpha$

$$
[u]_{\beta, K} \leq C\left(\alpha, \beta,\|f\|_{L^{\infty}(\Omega)},\|g\|_{L^{\infty}(\Omega)}, \operatorname{diam} \Omega, \operatorname{dist}(K, \partial \Omega)\right) .
$$

(ii) If $g \in C^{0, \beta}(\partial \Omega)$ for $0<\beta<\alpha$ then

$$
[u]_{\beta, \Omega} \leq C\left(\alpha, \beta,\|f\|_{L^{\infty}(\Omega)},[g]_{\beta, \partial \Omega}, \operatorname{diam} \Omega\right) .
$$

(iii) Assume that $f=0$. Then for each ball $B \subset \subset \Omega$

$$
[u]_{1, B} \leq C\left(\alpha,\|g\|_{L^{\infty}(\partial \Omega)}, \operatorname{diam} \Omega, \operatorname{dist}(B, \partial \Omega)\right) .
$$

(iv) If $f=0$ and $g \in C^{0, \alpha}(\partial \Omega)$ then

$$
[u]_{\alpha, \Omega}=[g]_{\alpha, \partial \Omega} .
$$

Remark 1.9. Part (iv) in Theorem 1.8 shows in particular that when $f=0$, the solution is an optimal Hölder extension of $g$ on $\Omega$. This is also the limit solution given by Theorem 1.1.

Remark 1.10. The uniqueness and the optimal $C^{0, \alpha}$-regularity of the solution remain open for general functions $f$.

Remark 1.11. Parts of Theorem 1.5 remain true when the distance $|x-y|^{\alpha}$ is replaced by a more general distance of the type $d(x-y)$, see Section 12.2 .

\section{Organization of the PAPER}

The structure of the paper is as follows: in Section 3 we try to make ourselves familiar with the operator $L$ and study some continuity properties of $L$ which later, in Section 4 , motivates the introduction of the notion of viscosity solutions. In Section 5 we give a representation formula of the solution in the case $f=0$. In Section 6 we prove Theorem 1.1. In Section 7 we prove a stability result, showing that certain limits of viscosity subsolutions are again viscosity subsolutions. In Section 8 we construct barriers, that we use later in Section 9, where we prove the existence of continuous solutions via Perron's method. In Section 10 we prove several regularity results of the solutions. In the end we also give the proof of Theorem 1.8. In Section 11 we prove a comparison principle in the case $f=0$. Using this we can conclude the proof of Theorem 1.5. In Section 12.2 we mention some possible generalizations of the problem and also some open questions that can be of general interest. 


\section{BASIC PROPERTIES OF $L$}

Here we present some properties of the operator $L$, which is clearly not well defined for all functions. Define

$$
\left(L^{+} u\right)(x)=\sup _{y \in \bar{\Omega}, y \neq x} \frac{u(y)-u(x)}{|y-x|^{\alpha}}, \quad\left(L^{-} u\right)(x)=\inf _{y \in \bar{\Omega}, y \neq x} \frac{u(y)-u(x)}{|y-x|^{\alpha}} .
$$

Lemma 3.1 (half relaxed limits for $L^{+}$and $L^{-}$). Consider a function $u: \bar{\Omega} \rightarrow \mathbb{R}$ and also a sequence of functions $\left(u_{\varepsilon}\right)_{\varepsilon}$ with $u_{\varepsilon}: \bar{\Omega} \rightarrow \mathbb{R}$ such that

$$
\left|u_{\varepsilon}-u\right|_{L^{\infty}(\bar{\Omega})} \rightarrow 0 \quad \text { as } \quad \varepsilon \rightarrow 0
$$

(i) If $u$ is upper semicontinuous, then

$$
\liminf _{\varepsilon \rightarrow 0}\left(L^{+} u_{\varepsilon}\right) \geq L^{+} u \quad \text { on } \quad \Omega
$$

(ii) If $u$ is lower semicontinuous, then

$$
\limsup _{\varepsilon \rightarrow 0} *\left(L^{-} u_{\varepsilon}\right) \leq L^{-} u \quad \text { on } \quad \Omega .
$$

Proof of Lemma 3.1. We give the proof of (3.1). The proof of (3.2) is similar. For any $x_{0} \in \Omega$ and $r>0$, let us set

where by definition, we have

$$
\left(L_{r}^{+} u\right)\left(x_{0}\right)=\sup _{y \in \bar{\Omega} \backslash B_{r}\left(x_{0}\right)} \frac{u(y)-u\left(x_{0}\right)}{\left|y-x_{0}\right|^{\alpha}}
$$

$$
\left(L^{+} u\right)\left(x_{0}\right)=\lim _{r \rightarrow 0}\left(L_{r}^{+} u\right)\left(x_{0}\right)=\sup _{r>0}\left(L_{r}^{+} u\right)\left(x_{0}\right) .
$$

Let us now consider a sequence $\left(x_{\varepsilon}\right)_{\varepsilon}$ of points of $\Omega$ such that $x_{\varepsilon} \rightarrow x_{0}$. For $\varepsilon$ small enough, we have $\left|x_{\varepsilon}-x_{0}\right|<$ $r / 2$, and then

$$
\left(L^{+} u_{\varepsilon}\right)\left(x_{\varepsilon}\right) \geq\left(L_{r / 2}^{+} u_{\varepsilon}\right)\left(x_{\varepsilon}\right)=\sup _{y \in \bar{\Omega} \backslash B_{r / 2}\left(x_{\varepsilon}\right)} \frac{u_{\varepsilon}(y)-u_{\varepsilon}\left(x_{\varepsilon}\right)}{\left|y-x_{\varepsilon}\right|^{\alpha}} \geq \sup _{y \in \bar{\Omega} \backslash B_{r}\left(x_{0}\right)} \frac{u_{\varepsilon}(y)-u_{\varepsilon}\left(x_{\varepsilon}\right)}{\left|y-x_{\varepsilon}\right|^{\alpha}} .
$$

Using that $-u$ is lower semicontinuous, we see that for any $y \in \bar{\Omega} \backslash B_{r}\left(x_{0}\right)$, we have

$$
\liminf _{\varepsilon \rightarrow 0}\left(L^{+} u_{\varepsilon}\right)\left(x_{\varepsilon}\right) \geq \frac{u(y)-u\left(x_{0}\right)}{\left|y-x_{0}\right|^{\alpha}}
$$

This implies

$$
\liminf _{\varepsilon \rightarrow 0}\left(L^{+} u_{\varepsilon}\right)\left(x_{\varepsilon}\right) \geq \sup _{y \in \bar{\Omega} \backslash B_{r}\left(x_{0}\right)} \frac{u(y)-u\left(x_{0}\right)}{\left|y-x_{0}\right|^{\alpha}} .
$$

Passing to the limit $r \rightarrow 0$, we deduce

$$
\liminf _{\varepsilon \rightarrow 0}\left(L^{+} u_{\varepsilon}\right)\left(x_{\varepsilon}\right) \geq \sup _{y \in \bar{\Omega}, y \neq x_{0}} \frac{u(y)-u\left(x_{0}\right)}{\left|y-x_{0}\right|^{\alpha}},
$$

for any sequence of points $x_{\varepsilon}$ converging to $x_{0}$. This shows (3.1).

This ends the proof of the lemma.

We then deduce immediately the following result. 
Definition 3.2 (semicontinuous envelopes). Consider a function $v: \bar{\Omega} \rightarrow \mathbb{R}$. Define

$$
v^{*}(x)=\limsup _{y \rightarrow x} v(y)
$$

and

$$
v_{*}(x)=\liminf _{y \rightarrow x} v(y) .
$$

The functions $v^{*}$ and $v_{*}$ are called the upper and lower semicontinuous envelopes of $v$.

Definition 3.3 (semicontinuity). We say that $v: \bar{\Omega} \rightarrow \mathbb{R}$ is upper semicontinuous (respectively lower semicontinuous) if $v^{*}=v$ (resp. $v_{*}=v$ ).

Corollary 3.4 (semicontinuity for $L^{+}$and $L^{-}$). Consider a function $u: \bar{\Omega} \rightarrow \mathbb{R}$.

(i) If $u$ is upper semicontinuous, then

$$
\left(L^{+} u\right)_{*}=L^{+} u \quad \text { on } \quad \Omega .
$$

(ii) If $u$ is lower semicontinuous, then

$$
\left(L^{-} u\right)^{*}=L^{-} u \text { on } \Omega .
$$

The following lemma motivates our choice of test functions when we later will define viscosity solutions.

Lemma 3.5 (continuity of $\left.L^{ \pm} \varphi\right)$. Let $\varphi \in C^{1}(\Omega)$. Then $L^{ \pm} \varphi \in C(\Omega)$.

Proof of Lemma 3.5. We only do the proof for $L^{+} \varphi$, the result for $L^{-} \varphi$ following from the equality $L^{-} \varphi=$ $-L^{+}(-\varphi)$. Take $x_{0} \in \Omega$.

Case (i): $\alpha \in(0,1)$

Then for $\delta$ small there exists a constant $C>0$ such that

$$
|\varphi(y)-\varphi(x)| \leq C|y-x| \text { for all } \quad x, y \in B_{\delta}\left(x_{0}\right) \subset \Omega .
$$

We recall the definition for $r>0$ of the operator for $x \in B_{\delta / 2}\left(x_{0}\right)$

$$
\left(L_{r}^{+} \varphi\right)(x)=\sup _{y \in \bar{\Omega} \backslash B_{r}(x)} \frac{\varphi(y)-\varphi(x)}{|y-x|^{\alpha}} .
$$

On one hand, by the continuity of $\varphi$, we see that $L_{r}^{+} \varphi$ is continuous on $\Omega$. On the other hand, we have for $r<\delta / 2$

$$
\left|\left(L^{+} \varphi\right)(x)-\left(L_{r}^{+} \varphi\right)(x)\right| \leq \sup _{y \in \Omega_{\cap} \cap B_{r}\left(x_{0}\right), y \neq x} \frac{|\varphi(y)-\varphi(x)|}{|y-x|^{\alpha}} \leq C r^{1-\alpha},
$$

which shows that the family $L_{r}^{+} \varphi$ of functions converges uniformly to $L \varphi$ as $r \rightarrow 0$ on $B_{\delta / 2}\left(x_{0}\right)$. This implies that $L^{+} \varphi$ is continuous.

Case (ii): $\alpha=1$

Fix $\delta>0$ such that $B_{\delta}\left(x_{0}\right) \subset \Omega$. Then there exists a modulus of continuity $\omega$ such that

$$
|\nabla \varphi(y)-\nabla \varphi(x)| \leq \omega(|y-x|) \quad \text { for all } \quad x, y \in B_{\delta}\left(x_{0}\right) .
$$

Using simply the formula for all $x, y \in B_{\delta}\left(x_{0}\right)$

$$
\varphi(y)-\varphi(x)=\int_{0}^{1} \mathrm{~d} t \nabla \varphi(x+t(y-x)) \cdot(y-x),
$$


we see that if furthermore $y \neq x$, then

$$
\left|\frac{\varphi(y)-\varphi(x)}{|y-x|}-\nabla \varphi(x) \cdot \frac{y-x}{|y-x|}\right| \leq \omega(|y-x|) .
$$

In particular if $x \in \overline{B_{\delta / 2}\left(x_{0}\right)}$, and $r \in(0, \delta / 2)$, then $B_{r}(x) \subset B_{\delta}\left(x_{0}\right) \subset \Omega$ and

$$
0 \leq \sup _{y \in \bar{\Omega} \cap B_{r}(x), y \neq x} \frac{\varphi(y)-\varphi(x)}{|y-x|}-|\nabla \varphi(x)| \leq \omega(r) .
$$

Remark that

$$
\left(L^{+} \varphi\right)(x)=\max \left(\left(L_{r}^{+} \varphi\right)(x), \quad \sup _{y \in \bar{\Omega} \cap B_{r}(x), y \neq x} \frac{\varphi(y)-\varphi(x)}{|y-x|}\right) .
$$

Now we have

$$
\left|\left(L^{+} \varphi\right)(x)-\left(L^{+} \varphi\right)\left(x_{0}\right)\right| \leq\left|\left(L_{r}^{+} \varphi\right)(x)-\left(L_{r}^{+} \varphi\right)\left(x_{0}\right)\right|+|| \nabla \varphi(x)|-| \nabla \varphi\left(x_{0}\right)||+2 \omega(r) .
$$

From the continuity of $L_{r}^{+} \varphi$ and $\nabla \varphi$, we deduce that

$$
\limsup _{x \rightarrow x_{0}}\left|\left(L^{+} \varphi\right)(x)-\left(L^{+} \varphi\right)\left(x_{0}\right)\right| \leq 2 \omega(r) .
$$

Choosing $r \rightarrow 0$, we deduce that

$$
\limsup _{x \rightarrow x_{0}}\left|\left(L^{+} \varphi\right)(x)-\left(L^{+} \varphi\right)\left(x_{0}\right)\right| \leq 0,
$$

and then $L^{+} \varphi$ is continuous at all points $x_{0} \in \Omega$.

This ends the proof of the lemma.

\section{Notion OF Viscosity SOLUtions}

We have seen how $L$ behaves when applied to sufficiently regular functions and we are now ready to introduce the notion of viscosity solutions. This notion follows the usual way of defining viscosity solutions. For a tour on the theory of viscosity solutions see [9]. For further reading on viscosity solutions of non-local operators, one can for instance consult [4].

Let

$$
L u=L^{+} u+L^{-} u
$$

when it is well defined, which indeed is the case for $u \in C^{1}(\Omega)$. We wish to study

$$
\begin{cases}L u=f & \text { in } \quad \Omega \\ u=g & \text { on } \quad \partial \Omega\end{cases}
$$

with $f \in C(\Omega)$ and $g \in C(\partial \Omega)$.

Definition 4.1 (viscosity sub/super/solution). Let $\alpha \in(0,1]$ and $f \in C(\Omega)$.

We say that $u$ is a subsolution (resp. supersolution) of (4.1) if $u$ is an upper semicontinuous (resp. lower semicontinuous) function from $\bar{\Omega}$ to $\mathbb{R}$ such that

(i) $u \leq g$ (resp. $u \geq g$ ) on $\partial \Omega$ 
(ii) for any test function $\varphi \in C^{1}(\Omega) \cap C(\bar{\Omega})$ satisfying

$$
u \leq \varphi \text { on } \bar{\Omega}(\text { resp. } u \geq \varphi)
$$

and $u\left(x_{0}\right)=\varphi\left(x_{0}\right)$ for some $x_{0} \in \Omega$, then

$$
(L \varphi)\left(x_{0}\right) \geq f\left(x_{0}\right)\left(\operatorname{resp} .(L \varphi)\left(x_{0}\right) \leq f\left(x_{0}\right)\right) .
$$

A function $u: \bar{\Omega} \rightarrow \mathbb{R}$ is a viscosity solution of (4.1), if and only if $u^{*}$ is a subsolution and $u_{*}$ is a supersolution.

We will say that a function $u: \bar{\Omega}: \rightarrow \mathbb{R}$ is a solution (resp sub- or supersolution) of (4.1) in $\Omega$ if $u$ only satisfies condition (ii) in Definition 4.1.

Remark 4.2. We see that this definition make sense intuitively, since if $u \in C^{1}(\Omega)$ and $\varphi \in C^{1}(\Omega) \cap \bar{\Omega}$ touches $u$ from above at $x_{0}$, we would indeed have

$$
(L \varphi)\left(x_{0}\right) \geq(L u)\left(x_{0}\right) .
$$

\section{A Representation Formula}

In the homogeneous case, i.e., when $f=0$, one can obtain an implicit representation of the solution, as presented in the following lemma.

Lemma 5.1 (representation formula when $f=0$ ). Let $\Omega$ be a bounded open set, $g \in C(\partial \Omega)$. Define for $x \in \Omega$ the non-increasing (in a) functions

$$
\ell_{x}^{+}(a)=\sup _{y \in \partial \Omega} \frac{g(y)-a}{|y-x|^{\alpha}}, \quad \ell_{x}^{-}(a)=\inf _{y \in \partial \Omega} \frac{g(y)-a}{|y-x|^{\alpha}}, \text { and } \quad \ell_{x}(a)=\ell_{x}^{+}(a)+\ell_{x}^{-}(a) .
$$

Then the function u defined by

$$
u(x)= \begin{cases}g(x) & \text { if } x \in \partial \Omega \\ a \quad \text { with } \quad \ell_{x}(a)=0 & \text { if } x \in \Omega\end{cases}
$$

is a solution of (4.1) which is continuous on $\bar{\Omega}$. Moreover, we have for all balls $B \subset \subset \Omega$, the estimate

$$
[u]_{1, B} \leq C\left(\alpha,\|g\|_{L^{\infty}(\partial \Omega)}, \operatorname{diam} \Omega, \operatorname{dist}(B, \partial \Omega)\right) .
$$

Before giving the proof of Lemma 5.1, we need the result below.

Lemma $5.2\left(|\cdot|^{\alpha}\right.$ is a distance). For $\alpha \in(0,1]$, the function $|\cdot|^{\alpha}$ is a distance, i.e.,

$$
|a+b|^{\alpha} \leq|a|^{\alpha}+|b|^{\alpha} .
$$

Proof of Lemma 5.2. The lemma follows from the observation that the function $f(r)=r^{\alpha}$ for $r \geq 0$ is concave and non-decreasing.

Proof of Lemma 5.1. We follow the ideas in [16]. From the definition of $u$, we deduce that

$$
\inf _{\partial \Omega} g \leq u(x) \leq \sup _{\partial \Omega} g \text { for all } x \in \Omega
$$

and then

$$
L_{x}^{-}:=\ell_{x}^{-}(u(x)) \leq 0 \leq \ell_{x}^{+}(u(x))=: L_{x}^{+} \quad \text { for all } \quad x \in \Omega .
$$


Step 1: first estimate when $L_{x_{1}}^{+} \leq L_{x_{2}}^{+}$

Let $x_{1}, x_{2} \in \Omega$ and let $x_{2}^{ \pm} \in \partial \Omega$ be such that

$$
g\left(x_{2}^{ \pm}\right)-u\left(x_{2}\right)=L_{x_{2}}^{ \pm}\left|x_{2}^{ \pm}-x_{2}\right|^{\alpha} .
$$

Then

$$
g\left(x_{2}^{+}\right)-u\left(x_{1}\right) \leq L_{x_{1}}^{+}\left|x_{2}^{+}-x_{1}\right|^{\alpha}
$$

This implies

$$
\begin{aligned}
u\left(x_{2}\right)-u\left(x_{1}\right) & \leq L_{x_{1}}^{+}\left|x_{2}^{+}-x_{1}\right|^{\alpha}-L_{x_{2}}^{+}\left|x_{2}^{+}-x_{2}\right|^{\alpha} \\
& \leq L_{x_{1}}^{+}\left(\left|x_{2}^{+}-x_{1}\right|^{\alpha}-\left|x_{2}^{+}-x_{2}\right|^{\alpha}\right) \\
& \leq L_{x_{1}}^{+}\left|x_{2}-x_{1}\right|^{\alpha},
\end{aligned}
$$

where we have used Lemma 5.2 and $L_{x_{1}}^{+} \geq 0$.

Step 2: second estimate when $L_{x_{1}}^{+} \leq L_{x_{2}}^{+}$

By the assumption on $L_{x_{i}}^{+}$we have $L_{x_{1}}^{-} \geq L_{x_{2}}^{-}$. Then

$$
g\left(x_{2}^{-}\right)-u\left(x_{1}\right) \geq L_{x_{1}}^{-}\left|x_{2}^{-}-x_{1}\right|^{\alpha}
$$

This implies

$$
\begin{aligned}
u\left(x_{2}\right)-u\left(x_{1}\right) & \geq L_{x_{1}}^{-}\left|x_{2}^{-}-x_{1}\right|^{\alpha}-L_{x_{2}}^{-}\left|x_{2}^{-}-x_{2}\right|^{\alpha} \\
& \geq L_{x_{2}}^{-}\left(\left|x_{2}^{-}-x_{1}\right|^{\alpha}-\left|x_{2}^{-}-x_{2}\right|^{\alpha}\right) \\
& \geq L_{x_{2}}^{-}\left|x_{2}-x_{1}\right|^{\alpha},
\end{aligned}
$$

where again we have used Lemma 5.2 and $L_{x_{1}}^{-} \leq 0$. This implies that

$$
u\left(x_{1}\right)-u\left(x_{2}\right) \leq-L_{x_{2}}^{-}\left|x_{2}-x_{1}\right|^{\alpha}=L_{x_{2}}^{+}\left|x_{2}-x_{1}\right|^{\alpha} .
$$

Step 3: estimate of $L^{+} u$

Adding the two steps above together, and interchanging the roles of $x_{1}$ and $x_{2}$ we have

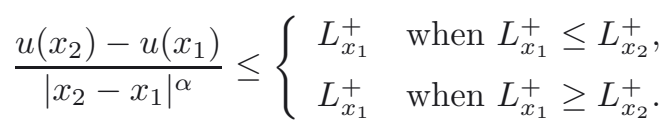

This implies $\left(L^{+} u\right)\left(x_{1}\right)=L_{x_{1}}^{+}$.

Step 4: estimate of $L^{-} u$

This can be done in a similar way as for $L^{+} u$.

\section{Step 5: pointwise solution}

Finally we get

$$
(L u)\left(x_{1}\right)=\ell_{x_{1}}\left(u\left(x_{1}\right)\right)=0
$$

which is true pointwise. In particular, this implies that $u$ is a viscosity solution of the equation. 


\section{Step 6: local continuity estimate for $u$}

Assume $b>a$ and take $a^{ \pm}$and $b^{ \pm}$such that

$$
\ell_{x}^{ \pm}(a)=\frac{g\left(a^{ \pm}\right)-a}{\left|x-a^{ \pm}\right|^{\alpha}}
$$

and similarly for $b$. Then

$$
\frac{b-a}{\left|x-b^{+}\right|^{\alpha}} \leq \frac{g\left(b^{+}\right)-a}{\left|x-b^{+}\right|^{\alpha}}-\frac{g\left(b^{+}\right)-b}{\left|x-b^{+}\right|^{\alpha}} \leq \ell_{x}^{+}(a)-\ell_{x}^{+}(b) .
$$

Hence,

$$
\ell_{x}^{+}(a)-\ell_{x}^{+}(b) \geq \frac{b-a}{(\operatorname{diam} \Omega)^{\alpha}} .
$$

After similar reasoning for $\ell_{x}^{-}$one can conclude (using the fact that $\ell_{x}^{ \pm}(a)$ is non-increasing in $a$ )

$$
\ell_{x}(a)-\ell_{x}(b) \geq \frac{2(b-a)}{(\operatorname{diam} \Omega)^{\alpha}}
$$

But for $x, y \in B \subset \subset \Omega$ we also have the inequality

$$
\begin{aligned}
\left|\ell_{x}(u(x))-\ell_{x}(u(y))\right| & \leq\left|\ell_{x}(u(x))-\ell_{y}(u(y))\right|+\left|\ell_{y}(u(y))-\ell_{x}(u(y))\right| \\
& \leq C\left(\alpha,|| g \|_{L^{\infty}(\partial \Omega)}, \operatorname{dist}(B, \partial \Omega)\right)|x-y| .
\end{aligned}
$$

Hence, with $b=\max (u(x), u(y))$ and $a=\min (u(x), u(y))$ in (5.2) we obtain

$$
|u(x)-u(y)| \leq \frac{(\operatorname{diam} \Omega)^{\alpha}}{2} C\left(\alpha,\|g\|_{L^{\infty}(\partial \Omega)}, \operatorname{dist}(B, \partial \Omega)\right)|x-y| .
$$

This implies (5.1).

Step 7: $u \in C(\bar{\Omega})$

It remains thus to prove that $u$ is continuous up to the boundary. Assume $x_{n} \rightarrow x_{0} \in \partial \Omega$ and let

$$
\ell_{x_{n}}^{ \pm}=\frac{g\left(y_{n}^{ \pm}\right)-u\left(x_{n}\right)}{\left|y_{n}^{ \pm}-x_{n}\right|^{\alpha}}
$$

for $y_{n}^{ \pm} \in \partial \Omega$. Since $\pm \ell_{x_{n}}^{ \pm} \geq 0$,

$$
g\left(y_{n}^{-}\right) \leq u\left(x_{n}\right) \leq g\left(y_{n}^{+}\right)
$$

We also know that

$$
\ell_{x_{n}}\left(u\left(x_{n}\right)\right)=0
$$

This implies that the limit of $\ell_{x_{n}}^{+}$is finite if and only if the limit of $\ell_{x_{n}}^{-}$is finite.

If they are both infinite then we must have $\left|y_{n}^{ \pm}-x_{n}\right| \rightarrow 0$. Using this in (5.3) together with the continuity of $g$ implies $u\left(x_{n}\right) \rightarrow g\left(x_{0}\right)=u\left(x_{0}\right)$.

If they are both finite then for some constant $C$

$$
C \geq \limsup _{n} \ell_{x_{n}}^{+} \geq \frac{g\left(x_{0}\right)-u\left(x_{n}\right)}{\left|x_{0}-x_{n}\right|^{\alpha}} \geq \liminf _{n} \ell_{x_{n}}^{-} \geq-C .
$$

This implies $u\left(x_{n}\right) \rightarrow u\left(x_{0}\right)$. This ends the proof of the lemma. 


\section{The Limit $p \rightarrow \infty$}

As mentioned in the introduction we will work with the so called fractional Sobolev space $W^{s, p}(\Omega)$. This space is equipped with the norm

$$
\|u\|_{W^{s, p}(\Omega)}=\|u\|_{L^{p}(\Omega)}+\left(\iint_{\Omega \times \Omega} \mathrm{d} x \mathrm{~d} y \frac{|u(x)-u(y)|^{p}}{|x-y|^{s p+N}}\right)^{\frac{1}{p}} .
$$

We recall the following result which can be found in [10], as Theorem 8.2.

Proposition 6.1 (Sobolev embedding). Let $u \in W^{s, p}(\Omega)$ for $s \in(0,1)$ and $s>N / p$ with $\Omega$ a bounded Lipschitz domain. Then with $\gamma=s-N / p$ we have

$$
\|u\|_{C^{0, \gamma}(\bar{\Omega})} \leq C\|u\|_{W^{s, p}(\Omega)} .
$$

\subsection{Proof of Theorem $\mathbf{1 . 1}$}

A key result throughout this section is the following convexity inequality.

Lemma 6.2 (convexity inequality). For $p \geq 1$, there holds

$$
|\min (a, c)-\min (b, d)|^{p}+|\max (a, c)-\max (b, d)|^{p} \leq|a-b|^{p}+|c-d|^{p} .
$$

For the sake of completeness we indicate a possible proof below. The idea is inspired by [15].

Proof of Lemma 6.2. The proof consists of, except in the obvious cases, observing that if $a>c$ and $b<d$ then there is $\theta$ such that

$$
c-b=\theta(a-b)+(1-\theta)(c-d), \quad a-d=(1-\theta)(a-b)+\theta(c-d),
$$

and using the convexity of the function $\phi(x)=|x|^{p}$. The case $a<c$ and $b>d$ can be treated in the exact same manner.

The lemma below justifies the existence and uniqueness of minimizers for $p$ large enough.

Lemma 6.3 (existence and uniqueness of a minimizer). Let $\alpha \in(0,1]$ and assume that $\Omega$ is a bounded Lipschitz domain. Consider $g \in C^{0, \alpha}(\partial \Omega)$ and define the set

$$
X_{g}=\{u \in C(\bar{\Omega}), \quad u=g \quad \text { on } \quad \partial \Omega\} .
$$

Define the minimization problem

$$
I=\inf _{u \in X_{g}} E_{p}(u)
$$

where

$$
E_{p}(u)=\int_{\Omega \times \Omega} \mathrm{d} x \mathrm{~d} y\left|\frac{u(x)-u(y)}{|x-y|^{\alpha}}\right|^{p} .
$$

Then for any $p>2 N / \alpha$, problem (6.1) has a unique minimizer $u_{p}$. Moreover, for any function $\varphi \in C_{c}^{\infty}(\Omega)$, we have

$$
\int_{\Omega \times \Omega} \mathrm{d} x \mathrm{~d} y\left|\frac{u_{p}(y)-u_{p}(x)}{|y-x|^{\alpha}}\right|^{p-1}\left\{\frac{\operatorname{sgn}\left(u_{p}(y)-u_{p}(x)\right)}{|y-x|^{\alpha}}\right\}(\varphi(y)-\varphi(x))=0 .
$$


Proof of Lemma 6.3. We first remark that there is $h \in X_{g}$ such that $E_{p}(h)<\infty$ which shows that $I<\infty$. Indeed, we can take one of the extensions from $[13,17]$

$$
h(x)=\sup _{y \in \partial \Omega}\left(g(y)-[g]_{\alpha, \partial \Omega}|x-y|^{\alpha}\right) \in C^{0, \alpha}(\bar{\Omega})
$$

Let us now consider a minimizing sequence $\left(u_{n}\right)_{n}$. We claim that we can assume $\left|u_{n}\right| \leq\|g\|_{L^{\infty}(\partial \Omega)}$. Indeed, we have by Lemma 6.2

$$
E_{p}\left(\max \left(u_{n},\|g\|_{L^{\infty}(\partial \Omega)}\right)\right)+E_{p}\left(\min \left(u_{n},\|g\|_{L^{\infty}(\partial \Omega)}\right)\right) \leq E_{p}\left(u_{n}\right)
$$

and also $\min \left(u_{n},\|g\|_{L^{\infty}(\partial \Omega)}\right) \in X_{g}$. In the same way we can show that the energy decreases if we cut $u_{n}$ from below at $-\|g\|_{L^{\infty}(\partial \Omega)}$. Hence, we can assume $\left|u_{n}\right| \leq\|g\|_{L^{\infty}(\partial \Omega)}$.

In addition,

$$
E_{p}\left(u_{n}\right) \leq|\Omega|^{2}\left([h]_{\alpha, \Omega}\right)^{p} \leq C\left(\alpha,[g]_{\alpha, \partial \Omega}\right) .
$$

From Proposition 6.1, we deduce that

$$
\left\|u_{n}\right\|_{C^{0, \gamma}(\bar{\Omega})} \leq C\left(\left(E_{p}\left(u_{n}\right)\right)^{\frac{1}{p}}+|\Omega|\|g\|_{L^{\infty}(\partial \Omega)}\right) \leq C\left(\alpha,[g]_{\alpha, \partial \Omega},\|g\|_{L^{\infty}(\partial \Omega)}\right)
$$

for $\gamma=\alpha-\frac{2 N}{p}>0$. Therefore, up to the extraction of a subsequence, we deduce that $u_{n}$ converges to a limit $u_{p}$ in $C^{0, \beta}(\bar{\Omega})$ for $\beta<\gamma$. As a consequence we have $u_{p} \in X_{g}$. Since the integrand converges a.e. it follows by Fatou's Lemma that $u_{p}$ is a minimizer. The uniqueness follows from the strict convexity of the functional and the fact that $u_{p}$ satisfies the corresponding Euler-Lagrange equation follows by perturbing with a test function in a standard way.

Now we will prove that minimizers are actually viscosity solutions, without knowing any regularity of the minimizer except continuity. For an example where a similar result is proved see [7].

Proposition 6.4 (minimizers are viscosity solutions). Let $p>2 \alpha / N$ and if $\alpha=1$ let $N \geq 2$. Then the minimizer of $E_{p}$ is a viscosity solution of the equation

$$
L_{p} u(x)=\int_{\Omega}\left|\frac{u(y)-u(x)}{|y-x|^{\alpha}}\right|^{p-1} \frac{\operatorname{sgn}(u(y)-u(x))}{|y-x|^{\alpha}} \mathrm{d} y=0 .
$$

Proof of Proposition 6.4. Take $u$ to be a minimizer of $E_{p}$. By Proposition 6.1, and the same arguments as in the proof of Lemma 6.3 we have $u \in C(\bar{\Omega})$. Now we need to prove that $u$ satisfies the viscosity inequality. We prove that $u$ is a subsolution.

Take $\varphi \in C^{1}(\Omega) \cap C(\bar{\Omega})$ touching $u$ from above at $x_{0} \in \Omega$. Then we want to show that $L_{p} \varphi\left(x_{0}\right) \geq 0$. Let

$$
\varphi^{\varepsilon}=\max (u, \varphi-\varepsilon)
$$

and

$$
\varphi_{\varepsilon}=\min (u, \varphi-\varepsilon)
$$

Up to replacing $\varphi(x)$ by $\varphi(x)+\delta\left|x-x_{0}\right|^{2}$, we see that for $\varepsilon$ small, we have $\varphi_{\varepsilon}=u$ and $\varphi^{\varepsilon}=\varphi-\varepsilon$ on $\partial \Omega$. Therefore $E_{p}\left(\varphi_{\varepsilon}\right) \geq E_{p}(u)$. Moreover, by Lemma 6.2

$$
E_{p}\left(\varphi^{\varepsilon}\right)+E_{p}\left(\varphi_{\varepsilon}\right) \leq E_{p}(u)+E_{p}(\varphi-\varepsilon)=E_{p}(u)+E_{p}(\varphi) .
$$

Consequently, $E_{p}\left(\varphi^{\varepsilon}\right) \leq E_{p}(\varphi)$. The convexity of $E_{p}$ then implies

$$
E_{p}\left((1-t) \varphi+t \varphi^{\varepsilon}\right) \leq(1-t) E_{p}(\varphi)+t E_{p}\left(\varphi^{\varepsilon}\right) \leq E_{p}(\varphi) .
$$


Consider the convex function

$$
f(t)=E_{p}\left(\varphi+t\left(\varphi^{\varepsilon}-\varphi\right)\right)
$$

Then we have

$$
\begin{aligned}
0 & \geq \frac{f(t)-f(0)}{t} \geq f^{\prime}(0) \\
& =\iint_{\Omega \times \Omega}\left|\frac{\varphi(x)-\varphi(y)}{|x-y|^{\alpha}}\right|^{p-1}\left(\frac{\operatorname{sgn}(\varphi(x)-\varphi(y))}{|x-y|^{\alpha}}\right)\left(\left(\varphi^{\varepsilon}-\varphi+\varepsilon\right)(x)-\left(\varphi^{\varepsilon}-\varphi+\varepsilon\right)(y)\right) \mathrm{d} y \mathrm{~d} x \\
& =2 \int_{\Omega}\left(\varphi^{\varepsilon}-\varphi+\varepsilon\right)(x)\left(\int_{\Omega}\left|\frac{\varphi(x)-\varphi(y)}{|x-y|^{\alpha}}\right|^{p-1}\left(\frac{\operatorname{sgn}(\varphi(x)-\varphi(y))}{|x-y|^{\alpha}}\right) \mathrm{d} y\right) \mathrm{d} x \\
& =2 \int_{\Omega}\left(\varphi^{\varepsilon}-\varphi+\varepsilon\right)(x)\left(-L_{p} \varphi\right)(x) \mathrm{d} x
\end{aligned}
$$

Now we argue by contradiction. If $L_{p} \varphi\left(x_{0}\right)<0$, then by continuity, which holds under our assumptions, because of Lebesgue's dominated convergence theorem, there is a small ball $B_{r}\left(x_{0}\right)$ such that $L_{p} \varphi<0$ in $B_{r}\left(x_{0}\right)$. Moreover, when $\varepsilon$ is small then $\operatorname{supp}\left(\varphi^{\varepsilon}-\varphi+\varepsilon\right) \subset B_{r}\left(x_{0}\right)$. We also observe that $\varphi^{\varepsilon} \geq \varphi-\varepsilon$ and in particular $\left(\varphi^{\varepsilon}-\varphi+\varepsilon\right)\left(x_{0}\right)=\varepsilon$. Hence, from the continuity of $u$, we see that there is a ball $B_{\delta}\left(x_{0}\right) \subset B_{r}\left(x_{0}\right)$ such that $\varphi^{\varepsilon}-\varphi+\varepsilon>0$ in $B_{\delta}\left(x_{0}\right)$. Therefore,

$$
\begin{aligned}
0 \geq \int_{\Omega}\left(\varphi^{\varepsilon}-\varphi+\varepsilon\right)(x)\left(-L_{p} \varphi\right)(x) \mathrm{d} x & =\int_{B_{r}\left(x_{0}\right)}\left(\varphi^{\varepsilon}-\varphi+\varepsilon\right)(x)\left(-L_{p} \varphi\right)(x) \mathrm{d} x \\
& \geq \int_{B_{\delta}\left(x_{0}\right)}\left(\varphi^{\varepsilon}-\varphi+\varepsilon\right)(x)\left(-L_{p} \varphi\right)(x) \mathrm{d} x>0,
\end{aligned}
$$

which is a contradiction.

In the same way it can be proved that $u$ is a viscosity supersolution.

To prove Theorem 1.1 we need the following technical result, whose proof is given in Section 6.2.

Lemma 6.5 (convergence of the $L^{p}$-norms). For $\varphi \in C^{1}(\Omega)$ let

$$
f_{p}(y)=\frac{\varphi(y)-\varphi\left(x_{p}\right)}{\left|y-x_{p}\right|^{\alpha}}
$$

and

$$
f(y)=\frac{\varphi(y)-\varphi\left(x_{0}\right)}{\left|y-x_{0}\right|^{\alpha}},
$$

where $x_{p} \rightarrow x_{0} \in \Omega$ as $p \rightarrow \infty$. If $\alpha=1$ assume in addition $N \geq 2$. Then

$$
\lim _{p \rightarrow \infty}\left\|\frac{f_{p}^{+}(y)}{\left|y-x_{p}\right|^{\frac{\alpha}{p}}}\right\|_{L^{p}(\Omega)}=\left\|f^{+}\right\|_{L^{\infty}(\Omega)},
$$

where $f_{p}^{ \pm}=\max \left( \pm f_{p}, 0\right)$. The same also holds for $f_{p}^{-}$.

Now we are ready to pass to the limit in the equation.

Proof of Theorem 1.1. Since

$$
E_{p}\left(u_{p}\right) \leq|\Omega|^{2}[h]_{\alpha, \Omega}^{p},
$$


we have with $q=2 N / \alpha+\delta$ for $\delta>0$ that

$$
E_{q}\left(u_{p}\right) \leq E_{p}^{\frac{q}{p}}|\Omega|^{\frac{2(p-q)}{p}} \leq[h]_{\alpha, \Omega}^{q}|\Omega|^{2} .
$$

By the same arguments as in the proof of Lemma 6.3 we can prove that $\left|u_{p}\right| \leq\|g\|_{L^{\infty}(\Omega)}$. Therefore, by Proposition 6.1, $u_{p}$ is uniformly bounded in $C^{0, \gamma}(\bar{\Omega})$ with $\gamma=\alpha-2 N / q>0$. Hence, for a subsequence, again labelled $u_{p}$, we have $u_{p} \rightarrow u$ in $C(\bar{\Omega})$.

Consider a test function $\varphi \in C^{1}(\Omega) \cap C(\bar{\Omega})$ such that

$$
\left\{\begin{array}{l}
u \leq \varphi \\
u\left(x_{0}\right)=\varphi\left(x_{0}\right) \text { for some } x_{0} \in \Omega,
\end{array}\right.
$$

and assume towards a contradiction that

$$
(L \varphi)\left(x_{0}\right)<0 .
$$

Up to replacing $\varphi$ by $\varphi+\delta\left|x-x_{0}\right|^{2}$ for $\delta$ small enough, we can furthermore assume that $x_{0}$ is a point of strict maximum of $u-\varphi$. Then

$$
\sup _{\bar{\Omega}}\left(u_{p}-\varphi\right)=\left(u_{p}-\varphi\right)\left(x_{p}\right)=M_{p}
$$

with

This shows that

$$
x_{p} \rightarrow x_{0}, \quad M_{p} \rightarrow 0 .
$$

$$
\left\{\begin{array}{l}
u_{p} \leq \varphi_{p}:=M_{p}+\varphi \\
u_{p}\left(x_{p}\right)=\varphi_{p}\left(x_{p}\right)
\end{array}\right.
$$

By Proposition 6.4, $u_{p}$ is a viscosity solution, therefore

$$
0 \leq\left(L_{p} \varphi_{p}\right)\left(x_{p}\right)=\left(L_{p} \varphi\right)\left(x_{p}\right)
$$

We recall that

$$
0 \leq\left(L_{p} \varphi\right)\left(x_{p}\right)=2 \int_{\Omega} \mathrm{d} y\left|\frac{\varphi(y)-\varphi\left(x_{p}\right)}{\left|y-x_{p}\right|^{\alpha}}\right|^{p-1}\left\{\frac{\operatorname{sgn}\left(\varphi(y)-\varphi\left(x_{p}\right)\right)}{\left|y-x_{p}\right|^{\alpha}}\right\} .
$$

Written in another way we have

$$
\left\|\left(\frac{\varphi(y)-\varphi\left(x_{p}\right)}{\left|y-x_{p}\right|^{\alpha+\frac{\alpha}{p-1}}}\right)^{+}\right\|_{L^{p-1}(\Omega)} \geq\left\|\left(\frac{\varphi(y)-\varphi\left(x_{p}\right)}{\left|y-x_{p}\right|^{\alpha+\frac{\alpha}{p-1}}}\right)^{-}\right\|_{L^{p-1}(\Omega)} .
$$

Lemma 6.5 now implies that we can pass to the limit in this inequality. Hence, we obtain

$$
\sup _{y \in \Omega}\left(\max \left(\frac{\varphi(y)-\varphi\left(x_{0}\right)}{\left|y-x_{0}\right|^{\alpha}}, 0\right)\right)+\inf _{y \in \Omega}\left(\min \left(\frac{\varphi(y)-\varphi\left(x_{0}\right)}{\left|y-x_{0}\right|^{\alpha}}, 0\right)\right) \geq 0 .
$$

Since $\varphi$ is $C^{1}$ at $x_{0}$ it is clear that $\pm\left(L^{ \pm} \varphi\right)\left(x_{0}\right) \geq 0$. Combined with the last inequality this implies,

$$
(L \varphi)\left(x_{0}\right) \geq 0,
$$

which contradicts (6.3). In the same way it can be proved that $u$ is a supersolution. 
By (ii) in Theorem 1.5 the solution $u$ is unique, so the whole sequence converges to the solution. Moreover, by (iv) in Theorem 1.8 we have

$$
[u]_{\alpha, \bar{\Omega}}=[g]_{\alpha, \partial \Omega}
$$

This ends the proof of the theorem.

\subsection{Proof of Lemma 6.5}

In order to prove Lemma 6.5 we first need the following result.

Lemma 6.6. For $\varphi \in C^{1}(\Omega)$ let

and

$$
f_{p}(y)=\frac{\varphi(y)-\varphi\left(x_{p}\right)}{\left|y-x_{p}\right|^{\alpha}}
$$

where $x_{p} \rightarrow x_{0} \in \Omega$ as $p \rightarrow \infty$. In addition, let

$$
f(y)=\frac{\varphi(y)-\varphi\left(x_{0}\right)}{\left|y-x_{0}\right|^{\alpha}},
$$

$$
f_{p}^{ \pm}=\max \left( \pm f_{p}, 0\right)
$$

and assume

Then for any

$$
\sup _{\Omega} f^{+}>0
$$

$$
0<t<\sup _{\Omega} f^{+}
$$

there is a $p_{0}<\infty$ and $c>0$ such that

$$
\left|\left\{f_{p}^{+}>t\right\}\right|>c
$$

for all $p \geq p_{0}$. The same also holds for $f_{p}^{-}$.

Proof of Lemma 6.6. For $\alpha<1$ this is obvious since $f_{p}$ will be uniformly continuous and then also $f_{p}^{+}$. Therefore we treat only the case $\alpha=1$. By arguments identical to those in the proof of Lemma 3.5 one can prove that

$$
\sup _{\Omega} f_{p}^{+} \rightarrow \sup _{\Omega} f^{+}
$$

Since $t<\sup _{\Omega} f^{+}$, there is a sequence $z_{p}$ such that $f_{p}^{+}\left(z_{p}\right)>t+\varepsilon$ for $\varepsilon$ small enough. We split the proof into two cases.

Case 1: $z_{p} \rightarrow x_{0}$.

By Taylor expansion we have $t+\varepsilon / 2 \leq\left|\nabla \varphi\left(x_{0}\right)\right|$ for $p$ large enough. We also have for all $y$

$$
f_{p}(y) \geq \nabla \varphi\left(x_{0}\right) \cdot \frac{y-x_{p}}{\left|y-x_{p}\right|}-o_{\left|y-x_{p}\right|}(1)-o_{\left|x_{p}-x_{0}\right|}(1)
$$

Therefore, if we choose $p$ large enough and $y$ such that $o_{\left|y-x_{p}\right|}(1)+o_{\left|x_{p}-x_{0}\right|}(1)<\varepsilon / 4$ and

$$
\nabla \varphi\left(x_{0}\right) \cdot \frac{y-x_{p}}{\left|y-x_{p}\right|} \geq\left|\nabla \varphi\left(x_{0}\right)\right|-\varepsilon / 4
$$

then $f_{p}(y)>t$. Clearly, this set of $y$ :s has positive measure, independently of $p$, as long as $p$ is large enough.

Case 2: $z_{p} \rightarrow z \neq x_{0}$.

In this case, for $p$ large enough, there is a $\delta$ such that $f_{p}^{+}$is uniformly continuous in $B_{\delta}\left(z_{p}\right)$, uniformly also in $p$. Consequently there is $\delta^{\prime}$, independent of $p$, such that $f_{p}^{+}>t$ in $B_{\delta^{\prime}}\left(z_{p}\right)$. 
Proof of Lemma 6.5.

Case 1: $\sup _{\Omega} f^{+}>0$.

Take

and let

$$
0<t<\sup _{\Omega} f^{+}
$$

$$
A(t, p)=\left\{f_{p}^{+}>t\right\}
$$

By Lemma 6.6, for $p>p_{0},|A(t, p)|>c>0$ with $c$ independent of $p$. Therefore

$$
\int_{\Omega} \frac{\left(f_{p}^{+}\right)^{p}}{\left|y-x_{p}\right|^{\alpha}} \geq t^{p} \int_{A(t, p)} \frac{1}{\left|y-x_{p}\right|^{\alpha}} \geq \frac{c}{(\operatorname{diam}(\Omega))^{\alpha}} t^{p} .
$$

This implies

For the other side of the inequality we have

$$
\left\|\frac{f_{p}^{+}}{\left|y-x_{p}\right|^{\frac{\alpha}{p}}}\right\|_{L^{p}(\Omega)} \geq t\left(\frac{c}{(\operatorname{diam}(\Omega))^{\alpha}}\right)^{\frac{1}{p}} \rightarrow t .
$$

$$
\int_{\Omega} \frac{\left(f_{p}^{+}\right)^{p}}{\left|y-x_{p}\right|^{\alpha}} \leq \sup _{\Omega}\left(f_{p}^{+}\right)^{p} \int_{\Omega} \frac{1}{\left|y-x_{p}\right|^{\alpha}} \leq C \sup _{\Omega}\left(f_{p}^{+}\right)^{p} .
$$

Thus

$$
\left\|\frac{f_{p}^{+}}{\left|y-x_{p}\right|^{\frac{\alpha}{p}}}\right\|_{L^{p}(\Omega)} \leq C^{\frac{1}{p}} \sup _{\Omega} f_{p}^{+} \rightarrow \sup _{\Omega} f^{+},
$$

where we have used (6.4) for the convergence. All together we have

$$
t \leq \liminf _{p \rightarrow \infty}\left\|\frac{f_{p}^{+}}{\left|y-x_{p}\right|^{\frac{\alpha}{p}}}\right\|_{L^{p}(\Omega)} \leq \limsup _{p \rightarrow \infty}\left\|\frac{f_{p}^{+}}{\left|y-x_{p}\right|^{\frac{\alpha}{p}}}\right\|_{L^{p}(\Omega)} \leq \sup _{\Omega} f^{+},
$$

for all

This implies the desired result.

$$
0<t<\sup _{\Omega} f^{+}
$$

Case 2: $\sup _{\Omega} f^{+}=0$.

Then (6.5) implies the result.

\section{Limits OF VISCOSITY SOLUTIONS}

In this section we prove the result that says that limits of subsolutions are again subsolutions.

Proposition 7.1 (stability of subsolutions).

(i) Consider a family $\left(F_{\varepsilon}\right)_{\varepsilon}$ of sets $F_{\varepsilon}$ of subsolutions of (4.1) in $\Omega$ and define for any $x_{0} \in \bar{\Omega}$

$$
\bar{u}\left(x_{0}\right)=\limsup _{\varepsilon \rightarrow 0, x_{\varepsilon} \rightarrow x_{0}, u_{\varepsilon} \in F_{\varepsilon}} u_{\varepsilon}\left(x_{\varepsilon}\right),
$$

which we assume to be bounded from above. Then $\bar{u}$ is a subsolution of (4.1) in $\Omega$.

(ii) Moreover, in the special case where the sets $F_{\varepsilon}=F$ are independent of $\varepsilon$, then we have

$$
\bar{u}=\bar{v}^{*} \quad \text { with } \quad \bar{v}(x)=\sup _{u \in F} u(x) \quad \text { for all } \quad x \in \bar{\Omega} .
$$


In fact, we will only be using the second statement of this proposition, but we give the full result since it can be of general interest.

To prove the proposition, we will need the following:

Lemma 7.2 (perturbation by a small parabola). Let $\varphi \in C^{1}(\Omega)$ and define for some $x_{0} \in \Omega$ and $\delta \in \mathbb{R}$

$$
\bar{\varphi}(x)=\varphi(x)+\delta\left(x-x_{0}\right)^{2} .
$$

Then, with the notation $R=\operatorname{diam} \Omega$ we have

$$
|(L \bar{\varphi})(x)-(L \varphi)(x)| \leq 4|\delta| R^{2-\alpha} \quad \text { for every } \quad x \in \Omega .
$$

Proof of Lemma 7.2. Consider points $y, x \in \Omega \backslash\left\{x_{0}\right\}$. We deduce

$$
\left|\frac{\delta\left(y-x_{0}\right)^{2}-\delta\left(x-x_{0}\right)^{2}}{|y-x|^{\alpha}}\right|=|\delta|\left|\frac{y-x}{|y-x|^{\alpha}} \cdot\left(y+x-2 x_{0}\right)\right| \leq 2|\delta| R^{2-\alpha} .
$$

This implies for $x \in \Omega \backslash\left\{x_{0}\right\}$

and then

$$
\left|\left(L^{ \pm} \bar{\varphi}\right)(x)-\left(L^{ \pm} \varphi\right)(x)\right| \leq 2|\delta| R^{2-\alpha}
$$

This ends the proof of the lemma.

$$
|(L \bar{\varphi})(x)-(L \varphi)(x)| \leq 4|\delta| R^{2-\alpha} .
$$

Proof of Proposition \%.1.

Preliminary: $\bar{u}$ is upper semicontinuous

Consider a sequence $\left(x_{\varepsilon}\right)_{\varepsilon}$ such that $x_{\varepsilon} \rightarrow x_{0}$ as $\varepsilon \rightarrow 0$ and

$$
\bar{u}^{*}\left(x_{0}\right)=\lim _{\varepsilon \rightarrow 0} \bar{u}\left(x_{\varepsilon}\right) .
$$

In particular, for any $\delta>0$, there exists a point $x_{\delta}$ such that

$$
\bar{u}^{*}\left(x_{0}\right)-\delta \leq \bar{u}\left(x_{\delta}\right) \quad \text { and } \quad\left|x_{\delta}-x_{0}\right| \leq \delta .
$$

By the definition of $\bar{u}$, there exist a sequence $y_{\varepsilon}$ and a function $u_{\varepsilon} \in F$ such that for $\varepsilon=\varepsilon_{\delta}<\delta$ we have

$$
\bar{u}\left(x_{\delta}\right)-\delta \leq u_{\varepsilon_{\delta}}\left(y_{\varepsilon_{\delta}}\right) \text { and }\left|x_{\delta}-y_{\varepsilon_{\delta}}\right| \leq \delta .
$$

Therefore

$$
\bar{u}^{*}\left(x_{0}\right)-2 \delta \leq u_{\varepsilon_{\delta}}\left(y_{\varepsilon_{\delta}}\right), \quad\left|y_{\varepsilon_{\delta}}-x_{0}\right| \leq 2 \delta \quad \text { and } \quad \varepsilon_{\delta}<\delta .
$$

Since this is true for any $\delta>0$, this shows that

$$
\bar{u}^{*}\left(x_{0}\right) \leq \bar{u}\left(x_{0}\right)
$$

and then $\bar{u}=\bar{u}^{*}$.

\section{Part I: proof that $\bar{u}$ is a subsolution}

We argue by contradiction and assume that there exists $\varphi \in C^{1}(\Omega) \cap C(\bar{\Omega})$ such that

$$
\bar{u} \leq \varphi \text { on } \bar{\Omega}
$$

with $\bar{u}\left(x_{0}\right)=\varphi\left(x_{0}\right)$ and $(L \varphi)\left(x_{0}\right)<f\left(x_{0}\right)$ for some $x_{0} \in \Omega$. 


\section{Step I.1: reducing the problem to a point of strict maximum}

Let us set for $\delta>0$

$$
\bar{\varphi}(x)=\varphi(x)+\delta\left(x-x_{0}\right)^{2}
$$

such that $x_{0}$ is a point of strict maximum of $\bar{u}-\bar{\varphi}$. From Lemma 7.2 we deduce that

$$
(L \bar{\varphi})\left(x_{0}\right) \leq(L \varphi)\left(x_{0}\right)+4 \delta R^{2-\alpha}<f\left(x_{0}\right)
$$

if $\delta$ is chosen small enough.

Step I.2: coming back to the $\varepsilon$-problem

Let us choose a sequence $\varepsilon$ with $x_{\varepsilon}$ and $u_{\varepsilon}$ such that

$$
\bar{u}\left(x_{0}\right)=\lim _{\varepsilon \rightarrow 0} u_{\varepsilon}\left(x_{\varepsilon}\right)
$$

Then let us set

$$
M_{\varepsilon}:=\sup _{x \in \bar{\Omega}}\left(u_{\varepsilon}-\bar{\varphi}\right)(x)=\left(u_{\varepsilon}-\bar{\varphi}\right)\left(y_{\varepsilon}\right) \quad \text { with } \quad y_{\varepsilon} \in \bar{\Omega} .
$$

Because $x_{0}$ is a point of strict maximum of $\bar{u}-\bar{\varphi}$, it is classical to realize that $M_{\varepsilon} \rightarrow 0$ and $y_{\varepsilon} \rightarrow x_{0}$. Let us set

$$
\bar{\varphi}_{\varepsilon}(x)=M_{\varepsilon}+\bar{\varphi}
$$

Then we have

and

$$
u_{\varepsilon} \leq \bar{\varphi}_{\varepsilon}
$$

$$
u_{\varepsilon}\left(y_{\varepsilon}\right)=\bar{\varphi}_{\varepsilon}\left(y_{\varepsilon}\right)
$$

which implies $(L \bar{\varphi})\left(y_{\varepsilon}\right) \geq f\left(y_{\varepsilon}\right)$, where we have used the fact that $L \bar{\varphi}_{\varepsilon}=L \bar{\varphi}$.

Therefore, by letting $y_{\varepsilon} \rightarrow x_{0}$ we can conclude that $(L \bar{\varphi})\left(x_{0}\right) \geq f\left(x_{0}\right)$. A contradiction to (7.1).

Part II: proof that $\bar{u}=\bar{v}^{*}$ when $F_{\varepsilon}=F$

Step II.1: $\bar{u} \geq \bar{v}^{*}$

By definition we have

$$
\bar{u}\left(x_{0}\right)=\limsup _{\varepsilon \rightarrow 0, x_{\varepsilon} \rightarrow x_{0}, u_{\varepsilon} \in F} u_{\varepsilon}\left(x_{\varepsilon}\right) .
$$

Setting $x_{\varepsilon}=x_{0}$, we see in particular that $\bar{u} \geq \bar{v}$, and then $\bar{u}^{*} \geq \bar{v}^{*}$. Using the fact that $\bar{u}=\bar{u}^{*}$, we deduce that

$$
\bar{u} \geq \bar{v}^{*}
$$

Step II.2: $\bar{u} \leq \bar{v}^{*}$

Let us fix $x_{0} \in \bar{\Omega}$ and sequences $\left(x_{\varepsilon}\right)_{\varepsilon},\left(u_{\varepsilon}\right)_{\varepsilon}$ such that

$$
\bar{u}\left(x_{0}\right)=\lim _{\varepsilon \rightarrow 0} u_{\varepsilon}\left(x_{\varepsilon}\right) \quad \text { and } \quad x_{\varepsilon} \rightarrow x_{0} .
$$

In particular, for any $\delta>0$, there exist $\varepsilon_{\delta}$ such that

$$
\bar{u}\left(x_{0}\right)-\delta \leq u_{\varepsilon_{\delta}}\left(x_{\varepsilon_{\delta}}\right) \leq \bar{v}\left(x_{\varepsilon_{\delta}}\right) \quad \text { and } \quad\left|x_{\varepsilon_{\delta}}-x_{0}\right| \leq \delta .
$$

This implies that $\bar{v}^{*}\left(x_{0}\right) \geq \bar{u}\left(x_{0}\right)$, i.e.

$$
\bar{u} \leq \bar{v}^{*}
$$

\section{Step II.3: conclusion}

We conclude that $\bar{u}=\bar{v}^{*}$.

This ends the proof of the proposition. 


\section{BARRIERS}

In order the prove the existence of solutions we need barriers, i.e., sub- and supersolutions. This section is devoted to the construction of barriers.

Lemma 8.1 (fundamental supersolutions). Consider a bounded open set $\Omega$ such that $0 \in \partial \Omega$. We also choose $R>0$ such that

$$
\bar{\Omega} \subset \overline{B_{R}(0)}
$$

Then for $\alpha \in(0,1]$, the function

$$
\Psi(x):=|x|^{\alpha}
$$

satisfies

$$
0 \geq-\delta(x) \geq(L \Psi)(x) \quad \text { for } \quad x \in \Omega
$$

where when $\alpha \in(0,1)$, we can choose

$$
-\delta(x):=-1+\frac{\rho^{\alpha}-1}{(\rho-1)^{\alpha}}<0 \quad \text { with } \quad \rho=R /|x| .
$$

Proof of Lemma 8.1. We simply estimate $(L \Psi)(x)$ for every $x \in \Omega$. We first remark that

$$
\left(L^{-} \Psi\right)(x) \leq \liminf _{y \rightarrow 0} \frac{|y|^{\alpha}-|x|^{\alpha}}{|y-x|^{\alpha}}=-1 .
$$

On the other hand we have with $e=x /|x|$

$$
\begin{aligned}
\left(L^{+} \Psi\right)(x) & =\sup _{y \in \bar{\Omega}, y \neq x} \frac{|y|^{\alpha}-|x|^{\alpha}}{|y-x|^{\alpha}} \\
& =\sup _{z \in \bar{\Omega} /|x|, z \neq e} \frac{|z|^{\alpha}-1}{|z-e|^{\alpha}} \\
& \leq \sup _{z \in \mathbb{R}^{N}, 1<|z| \leq R /|x|} \frac{|z|^{\alpha}-1}{|z-e|^{\alpha}} \\
& =\sup _{1<r \leq R /|x|} \frac{r^{\alpha}-1}{(r-1)^{\alpha}},
\end{aligned}
$$

where we have used for the last line the fact that $|z-e| \geq|| z|-| e||$. Now we set

$$
g(r):=\frac{r^{\alpha}-1}{(r-1)^{\alpha}}
$$

and compute

$$
\begin{aligned}
g^{\prime}(r) & =\frac{\alpha r^{\alpha-1}(r-1)^{\alpha}-\left(r^{\alpha}-1\right) \alpha(r-1)^{\alpha-1}}{(r-1)^{2 \alpha}} \\
& =\frac{\alpha(r-1)^{\alpha-1}}{(r-1)^{2 \alpha}}\left\{r^{\alpha-1}(r-1)-\left(r^{\alpha}-1\right)\right\} \\
& =\frac{\alpha(r-1)^{\alpha-1}}{(r-1)^{2 \alpha}}\left\{1-r^{\alpha-1}\right\} .
\end{aligned}
$$


In particular for $r>1$, we get $g^{\prime}(r) \geq 0$ and moreover

$$
g^{\prime}(r)>0 \text { for } r>1 \text { if } \alpha \in(0,1) .
$$

This implies that

$$
\left(L^{+} \Psi\right)(x) \leq g(R /|x|)
$$

where $g(R /|x|) \leq g(\infty)=1$ and moreover $g(R /|x|)<1$ if $\alpha \in(0,1)$. Joint to (8.2), this proves the lemma.

Lemma 8.2 (fundamental strict supersolutions for $\alpha=1$ ). Let $\alpha=1$. Consider a bounded open set $\Omega$ such that $0 \in \partial \Omega$. For $\varepsilon>0$ we set

Then we have

$$
\Psi_{\varepsilon}(x):=|x|-\varepsilon|x|^{2}
$$

$$
0>-\varepsilon|x| \geq\left(L \Psi_{\varepsilon}\right)(x) \quad \text { for all } \quad x \in \Omega .
$$

Proof of Lemma 8.2. We proceed as earlier. We have

$$
\left(L^{-} \Psi_{\varepsilon}\right)(x) \leq \liminf _{y \rightarrow 0} \frac{|y|-\varepsilon|y|^{2}-\left(|x|-\varepsilon|x|^{2}\right)}{|y-x|}=-1+\varepsilon|x| .
$$

On the other hand we have with $e=x /|x|$

$$
\begin{aligned}
\left(L^{+} \Psi_{\varepsilon}\right)(x) & =\sup _{y \in \bar{\Omega}, y \neq x} \frac{|y|-\varepsilon|y|^{2}-\left(|x|-\varepsilon|x|^{2}\right)}{|y-x|} \\
& =\sup _{z \in \bar{\Omega} /|x|, z \neq e} \frac{|z|-\bar{\varepsilon}|z|^{2}-(1-\bar{\varepsilon})}{|z-e|} \\
& \leq \sup _{z \in \mathbb{R}^{N}, 1<|z|} \frac{\left(|z|-\bar{\varepsilon}|z|^{2}-(1-\bar{\varepsilon})\right)^{+}}{|| z|-| e||} \\
& \leq \sup _{1<r} \frac{r-1-\bar{\varepsilon}\left(r^{2}-1\right)}{r-1} \\
& =\sup _{1<r} 1-\bar{\varepsilon}(r+1) \\
& =1-2 \bar{\varepsilon}
\end{aligned}
$$

where in the second line we have set

$$
\bar{\varepsilon}=\varepsilon|x|
$$

and where in the third line, we have used the fact that $|z-e| \geq|| z|-| e||$. Joint to (8.4), this shows that

$$
\left(L^{+} \Psi_{\varepsilon}\right)(x)+\left(L^{-} \Psi_{\varepsilon}\right)(x) \leq-\varepsilon|x|<0
$$

which ends the proof of the lemma.

We see that the strict sub- or supersolutions we have constructed above are not uniformly strict as we approach the origin $x=0$. However, if we demand less regularity, it is possible to construct strict sub- and supersolutions that remain strict when approaching the origin. These sub- and supersolutions will be useful later. 
Lemma 8.3 (less regular strict subsolutions/supersolutions). Consider a bounded open set $\Omega$ such that $0 \in \partial \Omega$. For $0<\beta<\alpha \in(0,1]$, the function

satisfies

$$
\Psi(x):=|x|^{\beta}
$$

$$
-\delta(x) \geq(L \Psi)(x) \quad \text { for } \quad x \in \Omega
$$

where

$$
\delta(x)=C(\alpha, \beta)|x|^{\beta-\alpha}>0 .
$$

Proof of Lemma 8.3. We proceed with the same computations as in Lemma 8.1 and obtain

$$
\left(L^{-} \Psi\right)(x) \leq-|x|^{\beta-\alpha},
$$

and

Now let

$$
\left(L^{+} \Psi\right)(x) \leq|x|^{\beta-\alpha} \sup _{1<r} \frac{r^{\beta}-1}{(r-1)^{\alpha}} .
$$

$$
h(r)=\frac{r^{\beta}-1}{(r-1)^{\alpha}} .
$$

Clearly, $g \rightarrow 0$ when $r \rightarrow \infty$. So for $R$ large enough, $r \geq R$ implies $g(r)<1 / 2$.

Case 1: $\alpha \in(0,1)$

When $r \leq R$ we have

$$
h(r) \leq \frac{r^{\alpha}-1}{(r-1)^{\alpha}}=g(r) \leq g(R)<1,
$$

where we have used (8.3). Therefore,

$$
\left(L^{+} \Psi\right)(x)<\max (h(R), 1 / 2)|x|^{\beta-\alpha}<|x|^{\beta-\alpha} .
$$

Case 2: $\alpha=1$

We have $h(r) \rightarrow h(1)=\beta$ as $r \searrow 1$. Moreover, $h(r)<1$ for $r>1$. Therefore,

$$
\sup _{1<r<R} h(r)=C_{0}<1 \text {. }
$$

This implies

Hence finally, in both cases

$$
\left(L^{+} \Psi\right)(x)<\max \left(C_{0}, 1 / 2\right)|x|^{\beta-\alpha}<|x|^{\beta-\alpha} .
$$

$$
(L \Psi)(x) \leq-C(\alpha, \beta)|x|^{\beta-\alpha} .
$$

Lemma 8.4 (natural subsolutions/supersolutions with boundary conditions). Let $f \in C(\Omega) \cap L^{\infty}(\Omega)$ and $g \in C(\partial \Omega)$. For $\beta \in(0, \alpha), x_{0} \in \mathbb{R}^{N}$ and $a, b \in \mathbb{R}$, we define

$$
u_{x_{0}, a, b}(x)=a+b\left|x-x_{0}\right|^{\beta} .
$$

Furthermore, let

and define for all $x \in \bar{\Omega}$

$$
S^{ \pm}=\left\{\begin{array}{l}
u_{x_{0}, a, b} \text { for }\left(x_{0}, a, \pm b\right) \in(\partial \Omega) \times \mathbb{R} \times(0, \infty) \\
\text { such that } \\
\pm u_{x_{0}, a, \pm b} \geq \pm g \text { on } \partial \Omega \\
\pm L u_{x_{0}, a, \pm b} \leq \pm f \quad \text { in } \Omega
\end{array}\right\}
$$

$$
\underline{v}(x)=\inf _{u \in S^{+}} u(x), \quad \bar{v}(x)=\sup _{u \in S^{-}} u(x) .
$$


Then $\underline{v} \in C(\bar{\Omega})$ is a supersolution and $\bar{v} \in C(\bar{\Omega})$ is a subsolution of (4.1).

Moreover, we have

$$
\bar{v} \leq \underline{v} \text { on } \bar{\Omega}
$$

and

$$
\bar{v}=g=\underline{v} \quad \text { on } \quad \partial \Omega .
$$

Proof of Lemma 8.4. Let us show that $\bar{v}$ is a continuous subsolution satisfying (8.6), the proof being similar to show that $\underline{v}$ is a supersolution.

Step 1: $\bar{v}^{*}$ is a subsolution

From Lemma 8.3, we first deduce that for $x_{0} \in \partial \Omega$ if $a$ and $b$ are chosen properly, then $u_{x_{0}, a, b} \in S^{-}$. This shows that $S^{-} \neq \emptyset$. On the other hand if $u_{x_{0}, a, b} \in S^{-}$then

$$
a \leq g\left(x_{0}\right) \leq \sup _{\partial \Omega} g,
$$

which implies that for all $u \in S^{-}$we have

$$
u \leq \sup _{\partial \Omega} g
$$

Therefore, applying the stability result (Prop. 7.1), and setting $F_{\varepsilon}=S^{-}$, we know that

$$
\bar{u}(x)=\limsup _{\varepsilon \rightarrow 0, x_{\varepsilon} \rightarrow x, u_{\varepsilon} \in S^{-}} u_{\varepsilon}\left(x_{\varepsilon}\right)
$$

is a viscosity subsolution. Moreover we have $\bar{v}^{*}=\bar{u}$.

Step 2: $\bar{v} \geq g$ on $\partial \Omega$

For any $x_{0} \in \partial \Omega$, and any $\delta>0$, we see (using the continuity of $g$ ) that there exists $b_{\delta}>0$ large enough such that with $a_{\delta}=g\left(x_{0}\right)-\delta$ there holds

$$
u_{x_{0}, a_{\delta},-b_{\delta}} \leq g \quad \text { on } \quad \partial \Omega
$$

Therefore

$$
\bar{v}\left(x_{0}\right) \geq g\left(x_{0}\right)-\delta .
$$

Since this true for any $\delta>0$, this implies that

$$
\bar{v}\left(x_{0}\right) \geq g\left(x_{0}\right)
$$

and then

\section{Step 3: $\bar{v}_{*}=\bar{v}$ on $\bar{\Omega}$}

$$
\bar{v} \geq g \quad \text { on } \quad \partial \Omega .
$$

Let $x_{0} \in \bar{\Omega}$ and take a sequence of functions $\left(u_{\delta}\right)_{\delta}$ with $u_{\delta} \in S^{-}$such that

$$
\bar{v}\left(x_{0}\right)=\lim _{\delta \rightarrow 0} u_{\delta}\left(x_{0}\right) .
$$

Now consider a sequence $\left(x_{\varepsilon}\right)_{\varepsilon}$ of points $x_{\varepsilon} \in \bar{\Omega}$ such that

$$
\bar{v}_{*}\left(x_{0}\right)=\lim _{\varepsilon \rightarrow 0} \bar{v}\left(x_{\varepsilon}\right) .
$$

Then we have

$$
\bar{v}\left(x_{\varepsilon}\right) \geq u_{\delta}\left(x_{\varepsilon}\right)
$$

which implies

$$
\bar{v}_{*}\left(x_{0}\right) \geq u_{\delta}\left(x_{0}\right) .
$$


Taking now the limit $\delta \rightarrow 0$, we get

$$
\bar{v}_{*}\left(x_{0}\right) \geq \bar{v}\left(x_{0}\right)
$$

and then

Step 4: $\bar{v}^{*}=\bar{v}$ on $\bar{\Omega}$

$$
\bar{v}_{*}=\bar{v} .
$$

From (8.7), we deduce that for any $x_{0} \in \bar{\Omega}$, there exist a sequence $\left(y_{\varepsilon}\right)_{\varepsilon}$ of points $y_{\varepsilon} \in \bar{\Omega}$ such that $y_{\varepsilon} \rightarrow x_{0}$ and a sequence $\left(u_{\varepsilon}\right)_{\varepsilon}$ of functions $u_{\varepsilon} \in S^{-}$such that

$$
\bar{v}^{*}\left(x_{0}\right)=\lim _{\varepsilon \rightarrow 0} u_{\varepsilon}\left(y_{\varepsilon}\right)
$$

We write

$$
u_{\varepsilon}(x)=u_{x_{\varepsilon}, a_{\varepsilon},-b_{\varepsilon}}(x)=a_{\varepsilon}-b_{\varepsilon}\left|x-x_{\varepsilon}\right|^{\beta}
$$

with $a_{\varepsilon} \in \mathbb{R}, b_{\varepsilon} \in(0, \infty), x_{\varepsilon} \in \partial \Omega$.

Case $b_{\varepsilon} \rightarrow \infty$

Since $a_{\varepsilon} \leq \sup _{\Omega} g$ and $\bar{v}^{*}\left(x_{0}\right) \leq \sup _{\Omega} g$ we deduce that $\left|y_{\varepsilon}-x_{\varepsilon}\right| \rightarrow 0$ which shows that

$$
x_{\varepsilon} \rightarrow x_{0} \quad \text { and } \quad x_{0} \in \partial \Omega \text {. }
$$

On the other hand we have $u_{\varepsilon}\left(x_{\varepsilon}\right) \leq g\left(x_{\varepsilon}\right)$ which means

$$
a_{\varepsilon} \leq g\left(x_{\varepsilon}\right)
$$

Therefore

$$
u_{\varepsilon}\left(y_{\varepsilon}\right) \leq a_{\varepsilon} \leq g\left(x_{\varepsilon}\right)
$$

Passing to the limit as $\varepsilon$ goes to zero, and using the continuity of $g$, we deduce from (8.9) that

$$
\bar{v}^{*}\left(x_{0}\right) \leq g\left(x_{0}\right) \leq \bar{v}\left(x_{0}\right)
$$

where we have used (8.8) for the last inequality. This shows in that case that

$$
\bar{v}^{*}\left(x_{0}\right) \leq \bar{v}\left(x_{0}\right)
$$

\section{Case $b_{\varepsilon}$ bounded}

Because of (8.9), we see that $a_{\varepsilon}$ is bounded. Then up to extraction of a subsequence, we can assume the following:

Therefore, with

$$
\left\{\begin{array}{l}
a_{\varepsilon} \rightarrow a \in \mathbb{R}, \\
b_{\varepsilon} \rightarrow b \in[0, \infty), \\
x_{\varepsilon} \rightarrow \bar{x}_{0} .
\end{array}\right.
$$

we get

$$
u_{0}=u_{\bar{x}_{0}, a,-b}
$$

$$
\bar{v}^{*}\left(x_{0}\right)=u_{0}\left(x_{0}\right) \leq \bar{v}\left(x_{0}\right)
$$

and then we conclude in every case that

$$
\bar{v}^{*}=\bar{v} \quad \text { on } \quad \bar{\Omega} .
$$




\section{Step 5: intermediate conclusion}

From the previous steps, we deduce that $\bar{v} \in C(\bar{\Omega})$ is a subsolution.

Step 6: proof of $\bar{v} \leq \underline{v}$ on $\bar{\Omega}$

Step 6.1: $u^{-} \leq u^{+}$

Let us consider $u^{+}=u_{x_{0}^{+}, a^{+}, b^{+}} \in S^{+}$and $u^{-}=u_{x_{0}^{-}, a^{-},-b^{-}} \in S^{-}$. By assumption we have

$$
u^{-} \leq g \leq u^{+} \quad \text { on } \quad \partial \Omega
$$

We want to show that

$$
u^{-} \leq u^{+} \text {on } \bar{\Omega} .
$$

Let us proceed by contradiction. If this is false, then we have

$$
0<\sup _{x \in \bar{\Omega}}\left(u^{-}-u^{+}\right)=\left(u^{-}-u^{+}\right)\left(y_{0}\right) \quad \text { for some point } \quad y_{0} \in \Omega
$$

and then

$$
\nabla\left(u^{-}-u^{+}\right)\left(y_{0}\right)=0
$$

i.e., for $x=y_{0}$

$$
\frac{\left(x-x_{0}^{+}\right)}{\left|x-x_{0}^{+}\right|} b^{+}\left|x-x_{0}^{+}\right|^{\beta-1}+\frac{\left(x-x_{0}^{-}\right)}{\left|x-x_{0}^{-}\right|} b^{-}\left|x-x_{0}^{-}\right|^{\beta-1}=0 .
$$

This implies that $y_{0} \in\left[x_{0}^{-}, x_{0}^{+}\right]$, because $b^{ \pm}>0$. Let us call $I=\left(z^{-}, z^{+}\right)$the connected component of $\left[x_{0}^{-}, x_{0}^{+}\right] \cap \Omega$ containing $y_{0}$. In particular since $\beta \in(0,1), u^{-}-u^{+}$is strictly convex on $I$ and reaches it maximum at the interior point $y_{0} \in I$. This gives immediately a contradiction.

\section{Step 6.2: conclusion}

From (8.12), we deduce that for any $x \in \bar{\Omega}$

$$
\bar{v}(x)=\sup _{u^{-} \in S^{-}} u^{-}(x) \leq u^{+}(x)
$$

and then

$$
\bar{v}(x) \leq \inf _{u^{+} \in S^{+}} u^{+}(x)=\underline{v}(x) .
$$

Therefore

$$
\bar{v} \leq \underline{v} \text { on } \bar{\Omega} .
$$

Step 7: proof of $\bar{v}=g=\underline{v}$ on $\partial \Omega$

Similarly to (8.8), we show that

$$
\underline{v} \leq g \text { on } \partial \Omega \text {. }
$$

Therefore from (8.13), we deduce that

$$
g \leq \bar{v} \leq \underline{v} \leq g \quad \text { on } \quad \partial \Omega
$$

and then

$$
\bar{v}=g=\underline{v} \quad \text { on } \quad \partial \Omega .
$$

This ends the proof of the lemma. 


\section{Perron's method}

In this section we construct the solutions applying the Perron's method.

Theorem 9.1 (existence by Perron's method). Let $u^{-} \in C(\bar{\Omega})$ be a subsolution (resp. $u^{+} \in C(\bar{\Omega})$ be a supersolution) of (4.1) with continuous boundary data $g$, satisfying

$$
\begin{cases}u^{-} \leq u^{+} & \text {on } \bar{\Omega}, \\ u^{-}=g=u^{+} & \text {on } \partial \Omega .\end{cases}
$$

Define

and for all $x_{0} \in \bar{\Omega}$

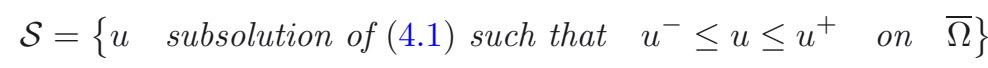

$$
\bar{u}\left(x_{0}\right)=\limsup _{\varepsilon \rightarrow 0, x_{\varepsilon} \rightarrow x_{0}, w_{\varepsilon} \in \mathcal{S}} w_{\varepsilon}\left(x_{\varepsilon}\right) .
$$

Then $\bar{u}$ is upper semicontinuous on $\bar{\Omega}$ and $\bar{u}$ is a viscosity solution of (4.1) in $\Omega$. Moreover, $\bar{u}$ satisfies

$$
u^{-} \leq \bar{u} \leq u^{+} \quad \text { on } \quad \bar{\Omega}
$$

Remark 9.2. From Lemma 8.4, we can set $u^{-}=\bar{u}$ and $u^{+}=\underline{u}$ and then Theorem 9.1 provides the existence of a solution.

\section{Proof of Theorem 9.1.}

\section{Step 1: construction of the maximal subsolution on $\bar{\Omega}$}

By assumption we have $\mathcal{S} \neq \emptyset$, because $u^{-} \in \mathcal{S}$. Applying the stability property of subsolutions (Prop. 7.1), we deduce that $\bar{u}$ is a subsolution on $\bar{\Omega}$. Finally, by construction, we get (9.1).

\section{Step 2: $\bar{u}_{*}$ is a supersolution on $\Omega$}

Let us proceed by contradiction and assume that $\bar{u}_{*}$ is not a supersolution on $\Omega$. Then there exists a test function $\varphi \in C^{1}(\Omega) \cap C(\bar{\Omega})$ and a point $x_{0} \in \Omega$ such that

$$
\left\{\begin{array}{l}
\bar{u}_{*} \geq \varphi \text { on } \bar{\Omega}, \\
\bar{u}_{*}\left(x_{0}\right)=\varphi\left(x_{0}\right) .
\end{array}\right.
$$

and $\bar{u}_{*}$ is not a supersolution at the point $x_{0}$, i.e.

$$
(L \varphi)\left(x_{0}\right)=\theta+f\left(x_{0}\right)>f\left(x_{0}\right) .
$$

Step 2.1: $\bar{u}_{*}\left(x_{0}\right)<u^{+}\left(x_{0}\right)$

We already know that $\bar{u} \leq u^{+}$on $\bar{\Omega}$, and then

$$
\varphi \leq \bar{u}_{*} \leq u^{+} \quad \text { on } \Omega
$$

If $\bar{u}_{*}\left(x_{0}\right)=u^{+}\left(x_{0}\right)$ and $x_{0} \in \Omega$, then $\varphi$ is a test function for $u^{+}$which is then in contradiction with the supersolution property of $u^{+}$at $x_{0}$. Therefore we have

$$
\bar{u}_{*}\left(x_{0}\right)<u^{+}\left(x_{0}\right) .
$$

\section{Step 2.2: preliminary}

Similarly to what was done in Step 1 of the proof of Proposition 7.1, we can set for $\delta>0$

$$
\varphi_{\delta}(x)=\varphi(x)-\delta\left|x-x_{0}\right|^{2} .
$$


From the result on perturbations by a small parabola (Lem. 7.2), we deduce that for $\delta>0$ small enough, the exists a radius $\rho>0$ such that

$$
\left(L \varphi_{\delta}\right) \geq \theta / 2+f>f \quad \text { on } \quad B_{\rho}\left(x_{0}\right) \subset \Omega .
$$

In particular, we see that $x_{0}$ is a point of strict minimum of $\bar{u}_{*}-\varphi_{\delta}$. We set for $\eta \geq 0$

$$
u_{\eta}(x)=\max \left(\bar{u}(x), \eta+\varphi_{\delta}(x)\right) .
$$

Let us consider a point $y_{0} \in \Omega$ and a test function $\psi \in C^{1}(\Omega) \cap C(\bar{\Omega})$ such that

$$
\left\{\begin{array}{l}
u_{\eta} \leq \psi \text { on } \bar{\Omega}, \\
u_{\eta}\left(y_{0}\right)=\psi\left(y_{0}\right) .
\end{array}\right.
$$

Step 2.3: $u_{\eta}$ is a subsolution on $\left\{u_{\eta}=\bar{u}\right\}$

Let us assume that $y_{0} \in\left\{u_{\eta}=\bar{u}\right\}$. Because $u_{\eta} \geq \bar{u}$, we deduce that $\psi$ is also a test function for $\bar{u}$ at $y_{0}$ and then $u_{\eta}$ satisfies the subsolution property at $y_{0}$ with the test function $\psi$.

Step 2.4: $u_{\eta}$ is a subsolution on $\left\{u_{\eta}>\bar{u}\right\} \cap \Omega$

When $\eta>0$, let us choose $r>0$ such that

$$
\eta=\delta r^{2} .
$$

This implies that

$$
\eta+\varphi_{\delta}(x) \leq \varphi(x) \leq \bar{u}_{*}(x) \leq \bar{u}(x) \quad \text { if } \quad x \notin B_{r}\left(x_{0}\right) \cap \bar{\Omega}
$$

and then

$$
\left\{u_{\eta}>\bar{u}\right\} \subset B_{r}\left(x_{0}\right) \subset B_{\rho}\left(x_{0}\right) \subset \Omega,
$$

if we choose $\eta$ small enough such that $r$ given by (9.6) satisfies

$$
r \leq \rho .
$$

Assume that $y_{0} \in\left\{u_{\eta}>\bar{u}\right\}$. Because $u_{\eta} \geq \eta+\varphi_{\delta}$, we deduce that $\psi$ is also a test function for $\eta+\varphi_{\delta}$ at $y_{0}$ and then

$$
(L \psi)\left(y_{0}\right) \geq\left(L\left(\eta+\varphi_{\delta}\right)\right)\left(y_{0}\right)=\left(L \varphi_{\delta}\right)\left(y_{0}\right) .
$$

From (9.5) and for the choice (9.7), we see that

$$
\left(L \varphi_{\delta}\right)\left(y_{0}\right) \geq \theta / 2+f\left(y_{0}\right)>f\left(y_{0}\right) .
$$

This shows that $u_{\eta}$ is a subsolution at $y_{0}$.

\section{Step 2.5: conclusion}

Therefore $u_{\eta}$ is a subsolution on $\bar{\Omega}$. On one hand we deduce from (9.4) that

$$
\eta+\varphi_{\delta} \leq u^{+} \quad \text { on } \bar{\Omega}
$$

for $\eta>0$ small enough, and then

$$
u^{-} \leq u_{\eta} \leq u^{+} \text {on } \bar{\Omega} .
$$

This shows that $u_{\eta} \in \mathcal{S}$ for $\eta>0$ small enough, and then $u_{\eta} \leq \bar{u}$. On the other hand, by definition of $\bar{u}_{*}$ there is a sequence of points $x_{\varepsilon} \rightarrow x_{0}$ such that

$$
\bar{u}_{*}\left(x_{0}\right)=\lim _{\varepsilon \rightarrow 0} \bar{u}\left(x_{\varepsilon}\right) \geq \lim _{\varepsilon \rightarrow 0} u_{\eta}\left(x_{\varepsilon}\right) \geq \lim _{\varepsilon \rightarrow 0} \eta+\varphi\left(x_{\varepsilon}\right)=\eta+\varphi\left(x_{0}\right)=\eta+\bar{u}_{*}\left(x_{0}\right),
$$


which is a contradiction. We finally conclude that $\bar{u}_{*}$ is a supersolution on $\Omega$, and then $\bar{u}$ is a viscosity solution on $\Omega$.

This ends the proof of the theorem.

\section{Regularity PROPERTIES}

\subsection{Continuity of subsolutions}

First out is the result that all subsolutions are actually continuous.

Proposition 10.1 (viscosity subsolutions are continuous). Let $f \in C(\Omega) \cap L^{\infty}(\Omega)$. If $u$ is a subsolution of (4.1) then $u \in C(\Omega)$.

Proof of Proposition 10.1. The proof is divided into several steps.

Step 1: discontinuity at $x_{0}$

We proceed by contradiction and assume that there exists a point $x_{0} \in \Omega$ and a sequence $\left(x_{\varepsilon}\right)_{\varepsilon}$ such that for some $\delta>0$

$$
u\left(x_{\varepsilon}\right) \leq u\left(x_{0}\right)-3 \delta \text { and } x_{\varepsilon} \rightarrow x_{0} .
$$

Because $u$ is upper semicontinuous, for each point $x_{\varepsilon}$, there exists $r_{\varepsilon}>0$ such that

$$
u \leq u\left(x_{\varepsilon}\right)+\delta \leq u\left(x_{0}\right)-2 \delta \quad \text { on } \quad B_{r_{\varepsilon}}\left(x_{\varepsilon}\right)
$$

\section{Step 2: construction of a first test function $\varphi$}

Because $u$ is upper semicontinuous, for any $\eta>0$, there exists $\rho_{\eta} \in(0,1)$ such that

$$
u<u\left(x_{0}\right)+\eta \quad \text { on } \quad B_{2 \rho_{\eta}}\left(x_{0}\right) \subset \Omega .
$$

Consider a test function $\varphi \in C^{1}(\bar{\Omega})$ satisfying

$$
\left\{\begin{array}{l}
\varphi=u\left(x_{0}\right)+\eta \quad \text { on } \quad B_{\rho_{\eta}}\left(x_{0}\right) \subset \subset \Omega \\
\varphi \geq u \text { on } \bar{\Omega} .
\end{array}\right.
$$

Step 3: the first perturbed test function

Let us now consider a function $\psi$ satisfying

$$
\left\{\begin{array}{l}
\psi \in C^{1}(\mathbb{R}), \\
0 \leq \psi(-z)=\psi(z) \leq 2, \\
\psi=0 \text { on } \quad \mathbb{R} \backslash[-1,1], \\
\psi(1 / 2)=1, \\
\psi^{\prime}<0 \text { on }(0,1), \\
\psi(0)=2 .
\end{array}\right.
$$

Put

$$
\Psi(x)=\psi(|x|) \quad \text { and } \quad M=\sup _{x \in \mathbb{R}^{N}}|\nabla \Psi(x)|,
$$


and define for $\lambda>0$

$$
\Psi_{x_{\varepsilon}}^{\lambda}=\Psi\left(\left(x-x_{\varepsilon}\right) / \lambda\right)
$$

Choosing the sequence $r_{\varepsilon}$ such that $r_{\varepsilon} \rightarrow 0$ as $\varepsilon \rightarrow 0$, we know that for $\varepsilon$ small enough we have

$$
B_{r_{\varepsilon}}\left(x_{\varepsilon}\right) \subset B_{\rho_{\eta} / 4}\left(x_{0}\right) .
$$

We then define

$$
u_{\varepsilon}^{\lambda}=u\left(x_{0}\right)+\eta-\eta \Psi_{x_{\varepsilon}}^{\lambda}
$$

and we set

$$
\lambda_{\varepsilon}=\sup A_{\varepsilon} \quad \text { with } \quad A_{\varepsilon}:=\left\{\lambda>0, \quad u<u_{\varepsilon}^{\lambda} \quad \text { on } \quad B_{\rho_{\eta}}\left(x_{0}\right)\right\}
$$

From (10.1), we deduce that if $\lambda \in\left(0, r_{\varepsilon}\right]$, then $\lambda \in A_{\varepsilon}$ if $\eta<\delta$. Moreover for

$$
\bar{\lambda}_{\varepsilon}=2\left|x_{\varepsilon}-x_{0}\right|
$$

we have $u_{\varepsilon}^{\bar{\lambda}_{\varepsilon}}\left(x_{0}\right)=u\left(x_{0}\right)$ and therefore $\bar{\lambda}_{\varepsilon} \notin A_{\varepsilon}$. Moreover we have $B_{\bar{\lambda}_{\varepsilon}}\left(x_{\varepsilon}\right) \subset B_{3\left|x_{\varepsilon}-x_{0}\right|}\left(x_{0}\right) \subset B_{3 \rho_{\eta} / 4}\left(x_{0}\right)$ because of (10.2). Therefore for any $0<\lambda \leq \bar{\lambda}_{\varepsilon}$, we have

$$
u_{\varepsilon}^{\lambda}=u\left(x_{0}\right)+\eta \quad \text { in a neighborhood of } \partial B_{\rho_{\eta}}\left(x_{0}\right) .
$$

Thus, there exists $\lambda_{\varepsilon} \in\left(r_{\varepsilon}, \bar{\lambda}_{\varepsilon}\right]$ and $y_{\varepsilon}$ such that

$$
\left\{\begin{array}{l}
u \leq u_{\varepsilon}^{\lambda_{\varepsilon}} \quad \text { on } \quad B_{\rho_{\eta}}\left(x_{0}\right), \\
u=u_{\varepsilon}^{\lambda_{\varepsilon}} \quad \text { at } \quad y_{\varepsilon} \in B_{\lambda_{\varepsilon}}\left(x_{\varepsilon}\right) \subset \subset B_{\rho_{\eta}}\left(x_{0}\right),
\end{array}\right.
$$

and due to $(10.1)$ we can see that $y_{\varepsilon} \notin B_{r_{\varepsilon}}\left(x_{\varepsilon}\right)$. See Figure 1 for a possible situation. We now define

$$
\varphi_{\varepsilon}(x)= \begin{cases}u_{\varepsilon}^{\lambda_{\varepsilon}} \geq u\left(x_{0}\right)-\eta & \text { if } \quad x \in B_{\rho_{\eta}}\left(x_{0}\right), \\ \varphi & \text { if } \quad x \in \bar{\Omega} \backslash B_{\rho_{\eta}}\left(x_{0}\right) .\end{cases}
$$

This can also be written as

$$
\varphi_{\varepsilon}=\varphi-\eta \Psi_{x_{\varepsilon}}^{\lambda_{\varepsilon}}
$$

Because of (10.3), we see that $\varphi_{\varepsilon} \in C^{1}(\bar{\Omega})$ and satisfies

$$
\left|\nabla \varphi_{\varepsilon}\right| \leq M \eta / \lambda_{\varepsilon} \quad \text { on } \quad B_{\lambda_{\varepsilon}}\left(x_{\varepsilon}\right) \subset \subset B_{\rho_{\eta}}\left(x_{0}\right)
$$




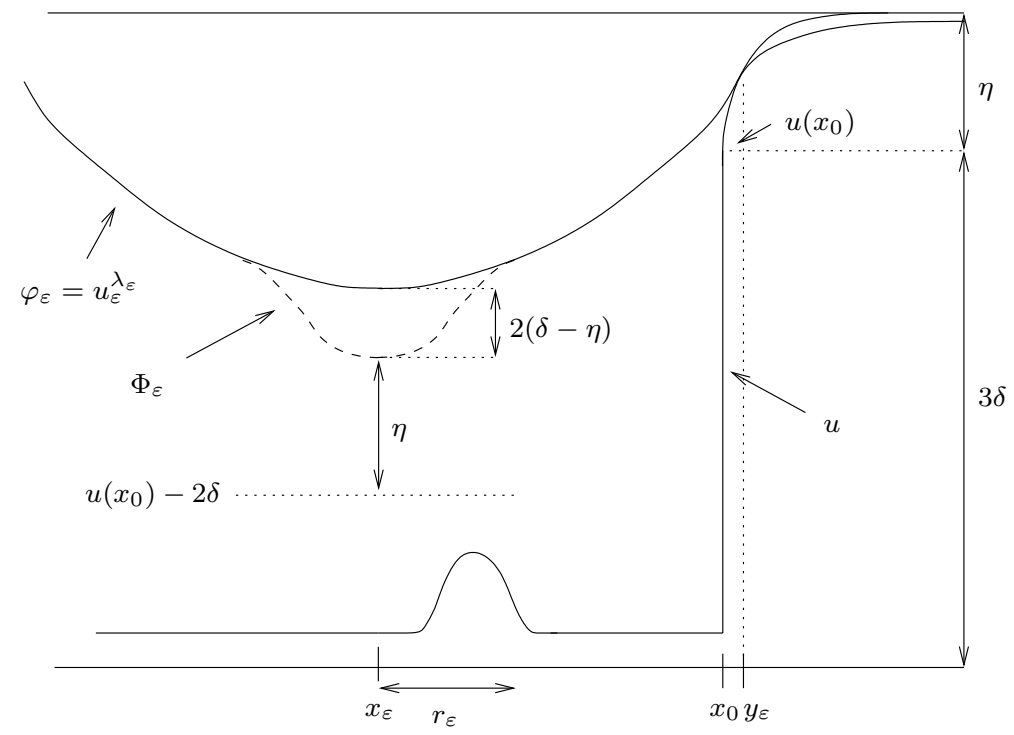

Figure 1. One possible situation of the chosen test functions.

and in particular we have with $\lambda_{\varepsilon} \leq \bar{\lambda}_{\varepsilon}=2\left|x_{\varepsilon}-x_{0}\right|<1 / 2$ for $\varepsilon$ small enough

$$
\begin{aligned}
\left(L^{+} \varphi_{\varepsilon}\right)\left(y_{\varepsilon}\right) & \leq \max \left(\sup _{y \in \bar{\Omega} \cap B_{\lambda_{\varepsilon}}\left(x_{\varepsilon}\right), y \neq y_{\varepsilon}} \frac{\varphi_{\varepsilon}(y)-\varphi_{\varepsilon}\left(y_{\varepsilon}\right)}{\left|y-y_{\varepsilon}\right|^{\alpha}}, \sup _{y \in \bar{\Omega} \backslash B_{\lambda_{\varepsilon}}\left(x_{\varepsilon}\right)} \frac{\varphi_{\varepsilon}(y)-\varphi_{\varepsilon}\left(y_{\varepsilon}\right)}{\left|y-y_{\varepsilon}\right|^{\alpha}}\right) \\
& \leq \max \left(\sup _{y \in \bar{\Omega} \cap B_{\lambda_{\varepsilon}}\left(x_{\varepsilon}\right), y \neq y_{\varepsilon}} \frac{-\eta \Psi_{x_{\varepsilon}}^{\lambda_{\varepsilon}}(y)+\eta \Psi_{x_{\varepsilon}}^{\lambda_{\varepsilon}}\left(y_{\varepsilon}\right)}{\left|y-y_{\varepsilon}\right|^{\alpha}}, \sup _{y \in \bar{\Omega} \backslash B_{\lambda_{\varepsilon}}\left(x_{\varepsilon}\right)} \frac{\varphi(y)-\varphi\left(y_{\varepsilon}\right)+\eta \Psi_{x_{\varepsilon}}^{\lambda_{\varepsilon}}\left(y_{\varepsilon}\right)}{\left|y-y_{\varepsilon}\right|^{\alpha}}\right) \\
& \leq \max \left(\frac{M \eta}{\left(\lambda_{\varepsilon}\right)^{\alpha}}, \quad\left(L^{+} \varphi\right)\left(y_{\varepsilon}\right)+\frac{\eta \Psi_{x_{\varepsilon}}^{\lambda_{\varepsilon}}\left(y_{\varepsilon}\right)}{\left(\lambda_{\varepsilon}\right)^{\alpha}}\right) \\
& \leq \max \left(\frac{2 M_{\eta}}{\left(\lambda_{\varepsilon}\right)^{\alpha}}, \quad\left(L^{+} \varphi\right)\left(y_{\varepsilon}\right)+\frac{2 \eta}{\left(\lambda_{\varepsilon}\right)^{\alpha}}\right) \\
& \leq \sup _{x \in \overline{B_{\rho_{\eta}}\left(x_{0}\right)}}\left(L^{+} \varphi\right)(x)+\frac{2 \bar{M} \eta}{\left(\lambda_{\varepsilon}\right)^{\alpha}} \quad \text { with } \bar{M}=\max (M, 1) .
\end{aligned}
$$

\section{Step 4: the second perturbed test function}

Define (with $\delta>\eta)$

$$
\Phi_{\varepsilon}=\varphi_{\varepsilon}-(\delta-\eta) \Psi_{x_{\varepsilon}}^{r_{\varepsilon}} \leq \varphi_{\varepsilon}
$$

which by (10.1) and (10.4) satisfies

$$
\left\{\begin{array}{l}
\Phi_{\varepsilon}\left(y_{\varepsilon}\right)=u\left(y_{\varepsilon}\right)=\varphi_{\varepsilon}\left(y_{\varepsilon}\right), \\
u \leq \Phi_{\varepsilon} .
\end{array}\right.
$$


Therefore

$$
\left(L^{+} \Phi_{\varepsilon}\right)\left(y_{\varepsilon}\right) \leq\left(L^{+} \varphi_{\varepsilon}\right)\left(y_{\varepsilon}\right) \leq \frac{\sup }{B_{1}\left(x_{0}\right)}\left(L^{+} \varphi\right)+\frac{2 \bar{M} \eta}{\left(\lambda_{\varepsilon}\right)^{\alpha}}
$$

Step 5: estimate of $L^{-} \Phi_{\varepsilon}$

We have

$$
\left(L^{-} \Phi_{\varepsilon}\right)\left(y_{\varepsilon}\right) \leq \frac{\Phi_{\varepsilon}\left(x_{\varepsilon}\right)-\Phi_{\varepsilon}\left(y_{\varepsilon}\right)}{\left|x_{\varepsilon}-y_{\varepsilon}\right|^{\alpha}}
$$

Using the fact that $\Phi_{\varepsilon}\left(x_{\varepsilon}\right)=u\left(x_{0}\right)+\eta-2 \delta, \Phi_{\varepsilon}\left(y_{\varepsilon}\right)=\varphi_{\varepsilon}\left(y_{\varepsilon}\right) \geq u\left(x_{0}\right)-\eta$ and (10.4), we get

$$
\left(L^{-} \Phi_{\varepsilon}\right)\left(y_{\varepsilon}\right) \leq-\frac{2(\delta-\eta)}{\left(\lambda_{\varepsilon}\right)^{\alpha}}
$$

\section{Step 6: conclusion}

For the choice $\eta<\delta /(\bar{M}+1)$ and using the fact that $\lambda_{\varepsilon} \rightarrow 0$ as $\varepsilon \rightarrow 0$, we see that

$$
\left(L \Phi_{\varepsilon}\right)\left(y_{\varepsilon}\right)=\left(L^{-} \Phi_{\varepsilon}\right)\left(y_{\varepsilon}\right)+\left(L^{+} \Phi_{\varepsilon}\right)\left(y_{\varepsilon}\right)<f\left(y_{\varepsilon}\right)
$$

for $\varepsilon$ small enough. This is in contradiction with the fact that $u$ is a subsolution.

This ends the proof of the proposition.

As a consequence we obtain the continuity of the solutions constructed by Perron's method.

Corollary 10.2. The solutions constructed in Theorem 9.1 are continuous on $\bar{\Omega}$.

Proof of Corollary 10.2. By the previous proposition, any subsolution and thus any solution is continuous inside $\Omega$. By construction, since the solutions is trapped between $u^{-}$and $u^{+}$, the solution is then also continuous up the boundary.

\subsection{Uniform regularity}

First we present a comparison result for certain sub- and supersolutions in "domains minus a point".

Lemma 10.3 (comparison). Let $x_{0} \in \Omega$ and assume that $u$ is upper semicontinuous and that in the viscosity sense there holds

with the boundary condition

$$
\left\{\begin{array}{l}
L u \geq f \quad \text { in } \Omega \\
L v<f \quad \text { in } \Omega \backslash\left\{x_{0}\right\}
\end{array}\right.
$$

If $v \in C^{1}\left(\Omega \backslash\left\{x_{0}\right\}\right) \cap C(\bar{\Omega})$, then $u \leq v$ in $\bar{\Omega}$.

$$
u \leq v \text { on } \partial \Omega \cup\left\{x_{0}\right\}
$$

Proof of Lemma 10.3. We argue by contradiction. If the assertion does not hold, then there is a point $y \in$ $\Omega \backslash\left\{x_{0}\right\}$ so that $u-v$ attains a positive maximum at $y$. If $v \in C^{1}(\Omega)$, $v$ will essentially be a test function for $u$ which gives a contradiction. If we assume only $v \in C^{1}\left(\Omega \backslash\left\{x_{0}\right\}\right)$ the result can be obtained by approximation.

We remark that due to this result combined with Lemma 8.3, we can compare solutions to "Hölder cones" of the type $C|x|^{\beta}$ for $\beta<\alpha<1$. Furthermore, if we are dealing with the homogeneous equations, we can take $\beta=\alpha$ due to Lemma 8.1 (and for $\alpha=1$ we had the special construction in Lem. 8.2).

Proposition 10.4 (bound in $\left.L^{\infty}\right)$. Let $f \in C(\Omega) \cap L^{\infty}(\Omega), g \in C(\partial \Omega)$ and $u$ be a viscosity solution of (4.1). Then there is $C\left(\alpha,\|g\|_{L^{\infty}(\partial \Omega)},\|f\|_{L^{\infty}(\Omega)}\right.$, diam $\left.\Omega\right)$ such that

$$
\|u\|_{L^{\infty}(\Omega)} \leq C
$$


Proof of Proposition 10.4. Fix $\beta \in(0, \alpha)$ and $x_{0} \in \partial \Omega$, and let

$$
v(x)=a+b\left|x-x_{0}\right|^{\beta},
$$

where

and $b$ is chosen so that

$$
a>\|g\|_{L^{\infty}(\partial \Omega)},
$$

$$
L v<-\|f\|_{L^{\infty}(\Omega)} .
$$

This is possible if $\beta<\alpha$ due to Lemma 8.3 , choosing $b$ such that

$$
-b C(\alpha, \beta)(\operatorname{diam} \Omega)^{\beta-\alpha}<-\|f\|_{L^{\infty}(\Omega)} .
$$

Then we are in the situation of Lemma 10.3 which implies $u^{*} \leq v$ in $\Omega$. Similarly we can obtain a bound from below.

Proposition 10.5 (partial regularity of solutions to the inhomogeneous equations). Let $f \in C(\Omega) \cap L^{\infty}(\Omega)$ and $u$ be a continuous viscosity solution of (4.1). Then for all $0<\beta<\alpha$, for all compact sets $K \subset \subset \Omega$ and with $d=\operatorname{dist}(K, \partial \Omega)$ we have

$$
[u]_{\beta, K} \leq \max \left(\frac{2\|u\|_{L^{\infty}(\Omega)}}{d^{\beta}}, \frac{\|f\|_{L^{\infty}(\Omega)}(\operatorname{diam} \Omega)^{\alpha-\beta}}{C(\alpha, \beta)}\right)
$$

where $C(\alpha, \beta)$ is defined in Lemma 8.3. If moreover, $g \in C^{0, \beta}(\partial \Omega)$. Then

$$
[u]_{\beta, \Omega} \leq \max \left(\|g\|_{C^{0, \beta}(\partial \Omega)}, \frac{\|f\|_{L^{\infty}(\Omega)}(\operatorname{diam} \Omega)^{\alpha-\beta}}{C(\alpha, \beta)}\right) .
$$

Proof of Proposition 10.5. For the first part, take $x_{0} \in K$ and

$$
v(x)=u\left(x_{0}\right)+\frac{C\|u\|_{L^{\infty}(\Omega)}}{d^{\beta}}\left|x-x_{0}\right|^{\beta}
$$

with $C \geq 2$ and so that $L v<f$ in $\Omega \backslash\left\{x_{0}\right\}$. This $C$ can be chosen uniformly with respect to the point $x_{0} \in K$. It is sufficient to assume

Then for $x \in \partial \Omega$ we have

$$
\frac{C\|u\|_{L^{\infty}(\Omega)}}{d^{\beta}} C(\alpha, \beta)(\operatorname{diam} \Omega)^{\beta-\alpha} \geq\|f\|_{L^{\infty}(\Omega)} .
$$

$$
u(x)-v(x)=u(x)-u\left(x_{0}\right)-\frac{C\|u\|_{L^{\infty}(\Omega)}}{d^{\beta}}\left|x-x_{0}\right|^{\beta} \leq 2\|u\|_{L^{\infty}(\Omega)}-\frac{C\|u\|_{L^{\infty}(\Omega)}}{d^{\beta}}\left|x-x_{0}\right|^{\beta} \leq 0 .
$$

Hence, by Lemma $10.3, u \leq v$ everywhere. Similarly we can obtain a bound from below of $u(x)-u\left(x_{0}\right)$. This concludes the first part.

For the second part, let $x_{0} \in \partial \Omega$ and

$$
v(x)=u\left(x_{0}\right)-C\left|x-x_{0}\right|^{\beta} .
$$

Clearly, $v(x) \leq u(x)$ for any $x \in \partial \Omega$ and $L v>f$ when $C$ is large enough (since $g \in C^{0, \alpha}$ and due to Lem. 8.3). Indeed, choose $C$ such that

$$
C \geq[g]_{\beta, \partial \Omega} \quad \text { and } \quad C C(\alpha, \beta)(\operatorname{diam} \Omega)^{\beta-\alpha} \geq\|f\|_{L^{\infty}(\Omega)}
$$


Thus, Lemma 10.3 implies $v(x) \leq u(x)$ for all $x \in \Omega$. Written differently, we have for any $x_{0} \in \partial \Omega$ and $x \in \Omega$,

$$
u\left(x_{0}\right) \leq u(x)+C\left|x-x_{0}\right|^{\beta}=w\left(x_{0}\right) .
$$

Thus, Lemma 10.3 applied with $w$ implies (becase of (10.5))

$$
u(y) \leq u(x)+C|x-y|^{\beta},
$$

for any $x, y \in \Omega$. Applying the same arguments to $-u$, implies a similar bound from below of $u(y)-u(x)$, and thus the proof is finished.

Proof of Theorem 1.8. Part (i) follows from Lemma 5.1, part (ii) follows from Proposition 10.5 and part (iii) follows from Proposition 10.5.

For part (iv), the result follows from the exact same arguments as in the proof of Proposition 10.5 with $\beta=\alpha$ and $C=[g]_{\alpha, \partial \Omega}$, using Lemmas 8.1 and 8.2. The reason why we can do this for the $\alpha$-barriers is simply that we do not need to compare with solutions having big or small operators $L$, since we are dealing with the homogeneous case.

Alternatively, one can apply the estimate in Proposition 10.5, taking $f=0$ and letting $\beta \rightarrow \alpha$.

Remark 10.6. As remarked by Luis Silvestre, when $f=0$, we obtain an optimal Hölder extension of $g$, for all exponents $\beta<\alpha$, and this holds also true for $\Delta_{\infty}$.

In fact, following the proof of Proposition 10.5, one realizes that something similar holds for a general operator $A$ (non-local or local) under quite mild assumptions on $A$, if we can find a strict supersolution (away from the origin) $v$ regular enough to be admissible as a test function such that

$$
v(x)=v(|x|), v(0)=0, v \geq 0 \text { and }\left|g(x)-g\left(x_{0}\right)\right| \leq C_{v} v\left(x-x_{0}\right) .
$$

Then if $A u=0$ in $\Omega$ and $u=g$ on $\partial \Omega$ there holds for all $x, x_{0} \in \Omega$

$$
\left|u(x)-u\left(x_{0}\right)\right| \leq C_{v} v\left(x-x_{0}\right) .
$$

\section{UNIQUENESS}

Finally we prove a uniqueness result under the same assumptions as in Lemma 5.1. The idea is to compare sub- and supersolutions to the solution given by the representation formula in Lemma 5.1, which then yields in the uniqueness.

Lemma 11.1 (convolution and Lipschitz with respect to the distance $\left.|\cdot|^{\alpha}\right)$. For $\alpha \in(0,1]$ assume that

$$
\frac{u(y)-u(x)}{|y-x|^{\alpha}} \leq L \quad \text { for all } \quad y, x \in B_{r}(0) .
$$

In addition, let $\rho_{\varepsilon}$ be a mollifier $\left(\int \rho_{\varepsilon}=1\right.$ and $\left.\rho_{\varepsilon} \geq 0\right)$ with support in $B_{\varepsilon}(0)$. Then $u_{\varepsilon}=\rho_{\varepsilon} \star u$ satisfies when $\varepsilon<r$

$$
\frac{u_{\varepsilon}(y)-u_{\varepsilon}(x)}{|y-x|^{\alpha}} \leq L \quad \text { for all } \quad y, x \in B_{r-\varepsilon}(0)
$$


Proof of Lemma 11.1. For all $x, y \in B_{r-\varepsilon}(0)$, we have with $y=x+h$

$$
\begin{aligned}
u_{\varepsilon}(x+h)-u_{\varepsilon}(x) & =\left(\rho_{\varepsilon} \star u\right)(x+h)-\left(\rho_{\varepsilon} \star u\right)(x) \\
& =\int \mathrm{d} z \rho_{\varepsilon}(z)\{u(x+h-z)-u(x-z)\} \\
& \leq \int \mathrm{d} z \rho_{\varepsilon}(z) L|h|^{\alpha} \\
& =L|h|^{\alpha} .
\end{aligned}
$$

This shows exactly the expected result.

Proposition 11.2 (comparison when $f=0$ ). Under the hypotheses of Lemma 5.1, take $u$ to be the therein implicitly defined solution and $v$ a subsolution (resp. a supersolution) of (4.1). Then $u \geq v$ (resp. $u \leq v$ ).

Proof of Proposition 11.2. We give the proof for the case when $v$ is a subsolution, the proof being similar when $v$ is a supersolution.

\section{Step 1: preliminaries}

We first observe that we can apply steps 1-4 of the proof of Lemma 5.1 to obtain

$$
\left(L^{ \pm} u\right)(x)=\ell_{x}^{ \pm}(u(x))
$$

Assume that

$$
M=\sup _{\bar{\Omega}}(v-u)>0
$$

and consider the set

$$
K_{0}=\{x \in \bar{\Omega}, v(x)-u(x)=M\} .
$$

Because $u \in C(\bar{\Omega})$ and $v$ is upper semi continuous, we see that the compact set $K_{0}$ satisfies

$$
K_{0} \subset \subset \Omega
$$

For some fixed $\delta>0$ small enough, let us consider a compact $\delta$-neighborhood $K_{\delta}^{+}$of $K_{0}$ satisfying

$$
K_{0} \subset \subset K_{\delta}^{+} \subset \subset \Omega
$$

and a $\delta$-neighborhood $\Omega_{\delta}$ of $\Omega$. We first extend $u$ on $\Omega_{\delta}$ by a continuous function still denoted by $u$. Since $u$ is continuous on $\bar{\Omega}$ this can be done thanks to a theorem of Lebesgue, found in [11]. In fact there is also an explicit extension

$$
u_{\mathrm{ext}}(x)=\inf _{y \in \bar{\Omega}}(\omega(x-y)+u(y))
$$

if $\omega$, the modulus of continuity of $u$ on $\bar{\Omega}$, is assumed to be continuous. If $\omega$ is a distance, then $u_{\text {ext }}$ is $C^{\omega}$-continuous, otherwise it might have a slighty worse modulus of continuity.

Then consider a mollifier $\rho_{\varepsilon}(x)$ and set

$$
u_{\varepsilon}=\rho_{\varepsilon} \star u
$$

and

$$
M_{\varepsilon}=\sup _{\bar{\Omega}}\left(v-u_{\varepsilon}\right) \geq M / 2>0
$$

where the bound from below holds for $\varepsilon$ small enough. Moreover we also have

$$
K_{\varepsilon}:=\left\{x \in \bar{\Omega}, v(x)-u_{\varepsilon}(x)=M_{\varepsilon}\right\} \subset \subset K_{\delta}^{+} \subset \subset \Omega
$$


for $\varepsilon$ small enough. We then deduce that

$$
\left\{\begin{array}{c}
v \leq M_{\varepsilon}+u_{\varepsilon}=: \varphi_{\varepsilon}, \\
v=\varphi_{\varepsilon} \text { on } K_{\varepsilon} .
\end{array}\right.
$$

On the other hand, by the upper semi continuity of $v$, there exists a neighborhood $V$ of $\partial \Omega$ in $\bar{\Omega}$ such that for $\varepsilon$ small enough

Let $\psi \in C^{\infty}\left(\mathbb{R}^{N}\right)$ such that

$$
v \leq u_{\varepsilon}+M / 8 \quad \text { on } \quad V \subset \bar{\Omega} \backslash K_{\delta}^{+} .
$$

$$
\left\{\begin{array}{l}
\psi=1 \quad \text { on } \quad \partial \Omega, \\
\psi=0 \text { on } \quad \Omega \backslash V, \\
0 \leq \psi \leq 1 \quad \text { on } \bar{\Omega} .
\end{array}\right.
$$

Then set

which satisfies

$$
\tilde{\varphi}_{\varepsilon}=\varphi_{\varepsilon}-\frac{M}{4} \psi
$$

$$
\left\{\begin{array}{l}
v \leq \tilde{\varphi}_{\varepsilon}, \\
v=\tilde{\varphi}_{\varepsilon} \text { on } K_{\varepsilon} .
\end{array}\right.
$$

Therefore for any $x_{\varepsilon} \in K_{\varepsilon}, \tilde{\varphi}_{\varepsilon}$ is a test function for $v$ at $x_{\varepsilon}$, and then

$$
0 \leq\left(L \tilde{\varphi}_{\varepsilon}\right)\left(x_{\varepsilon}\right)
$$

\section{Step 2: limit for $L^{-}$}

Up to extraction of a subsequence, we have $x_{\varepsilon} \rightarrow x_{0} \in \Omega$. Moreover $u_{\varepsilon}$ converges to $u$ uniformly on $\bar{\Omega}$, and then $M_{\varepsilon} \rightarrow M$. From Lemma 3.1 (ii), we deduce with $\tilde{\varphi}_{0}=M+u-\frac{M}{4} \psi$ that

$$
\begin{aligned}
\limsup _{\varepsilon \rightarrow 0}\left(L^{-} \tilde{\varphi}_{\varepsilon}\right)\left(x_{\varepsilon}\right) & \leq\left(L^{-} \tilde{\varphi}_{0}\right)\left(x_{0}\right) \\
& \leq\left(L^{-}\left(u-\frac{M}{4} \psi\right)\right)\left(x_{0}\right) \\
& \leq \inf _{y \in \partial \Omega} \frac{g(y)-M / 4-u\left(x_{0}\right)}{\left|y-x_{0}\right|^{\alpha}} \\
& \leq\left(L^{-} u\right)\left(x_{0}\right)-\delta_{0} \quad \text { with } \quad \delta_{0}=\frac{M}{4 \sup _{y \in \partial \Omega}\left|y-x_{0}\right|^{\alpha}} .
\end{aligned}
$$

\section{Step 3: limit for $L^{+}$}

We have

$$
\left(L^{+} \tilde{\varphi}_{\varepsilon}\right)\left(x_{\varepsilon}\right) \leq\left(L^{+} \varphi_{\varepsilon}\right)\left(x_{\varepsilon}\right)=\left(L^{+} u_{\varepsilon}\right)\left(x_{\varepsilon}\right)
$$

For any $x \in \Omega$ let us set

$$
L_{x}^{+}=\sup _{y \in \partial \Omega} \frac{g(y)-u(x)}{|y-x|^{\alpha}} .
$$

From the continuity of $u$, we deduce that the map $x \mapsto L_{x}^{+}$is continuous on $\Omega$. In particular for any $\eta>0$, there exists $r>0$ such that

$$
\left|L_{x}^{+}-L_{x_{0}}^{+}\right| \leq \eta \quad \text { for all } \quad x \in B_{r}\left(x_{0}\right) \subset \subset \Omega
$$


We also recall that due to (11.1), for all $x \in \Omega$ we have

$$
u(y)-u(x) \leq L_{x}^{+}|y-x|^{\alpha} \quad \text { for all } y \in \bar{\Omega}
$$

and then for all $x \in B_{r}\left(x_{0}\right)$

$$
u(y)-u(x) \leq\left(L_{x_{0}}^{+}+\eta\right)|y-x|^{\alpha} \quad \text { for all } y \in \bar{\Omega} .
$$

Up to choosing $\delta$ small enough, we can always assume that the extension $u$ on $\Omega_{\delta}$ satisfies for all $x \in B_{r}\left(x_{0}\right)$

$$
u(y)-u(x) \leq a|y-x|^{\alpha} \quad \text { for all } \quad y \in \Omega_{\delta} \quad \text { with } \quad a=\left(L_{x_{0}}^{+}+2 \eta\right)
$$

Lemma 11.1 implies for $\varepsilon$ small enough that

$$
u_{\varepsilon}(y)-u_{\varepsilon}(x) \leq a|y-x|^{\alpha} \quad \text { on } \quad B_{r / 2}\left(x_{0}\right)
$$

Now, choose $\varepsilon$ small enough such that $\left|x_{\varepsilon}-x_{0}\right|<r / 4$. Then we have

$$
\left(L^{+} u_{\varepsilon}\right)\left(x_{\varepsilon}\right) \leq \max \left(a, \sup _{y \in \bar{\Omega} \backslash B_{r / 4}\left(x_{\varepsilon}\right)} \frac{u_{\varepsilon}(y)-u_{\varepsilon}\left(x_{\varepsilon}\right)}{\left|y-x_{\varepsilon}\right|^{\alpha}}\right) .
$$

Therefore we deduce from the uniform convergence of $u_{\varepsilon}$ towards $u$ that

$$
\limsup _{\varepsilon \rightarrow 0}\left(L^{+} u_{\varepsilon}\right)\left(x_{\varepsilon}\right) \leq \max \left(a, \sup _{y \in \bar{\Omega} \backslash B_{r / 4}\left(x_{0}\right)} \frac{u(y)-u\left(x_{0}\right)}{\left|y-x_{0}\right|^{\alpha}}\right)=a=L_{x_{0}}^{+}+2 \eta .
$$

Since this is true for any $\eta>0$, we obtain

$$
\limsup _{\varepsilon \rightarrow 0}\left(L^{+} \tilde{\varphi}_{\varepsilon}\right)\left(x_{\varepsilon}\right) \leq \limsup _{\varepsilon \rightarrow 0}\left(L^{+} u_{\varepsilon}\right)\left(x_{\varepsilon}\right) \leq L_{x_{0}}^{+}=\left(L^{+} u\right)\left(x_{0}\right) .
$$

\section{Step 4: conclusion}

From (11.2)-(11.4), we deduce that

$$
\limsup _{\varepsilon \rightarrow 0}\left(L \tilde{\varphi}_{\varepsilon}\right)\left(x_{\varepsilon}\right) \leq(L u)\left(x_{0}\right)-\delta_{0}=0-\delta_{0} \quad \text { with } \quad \delta_{0}>0 .
$$

This is in contradiction with the property $L u=0$ satisfied by $u$ pointwisely.

This ends the proof.

Remark 11.3. In the proof above, the essential key is the fact that the supremum and the infimum in $L^{ \pm} u$ are attained on $\partial \Omega$ for the solution given by the representation formula in Lemma 5.1.

Proof of Theorem 1.5. Part (i) follows from Theorem 9.1, Remark 9.2 and Corollary 10.2, while part (ii) is an immediate consequence of Proposition 11.2.

\section{Generalizhtions}

\subsection{Replacing $\Omega$ with $\mathbb{R}^{n}$}

We remark here that we can replace $\Omega$ by $\mathbb{R}^{n}$ and instead consider the problem

$$
\left\{\begin{array}{l}
L_{\mathbb{R}^{n}} u=f \text { in } \Omega \\
u=g \text { in } \mathbb{R}^{n} \backslash \Omega
\end{array}\right.
$$


where

$$
L_{\mathbb{R}^{n}} u=\sup _{y \in \mathbb{R}^{n}, y \neq x} \frac{u(y)-u(x)}{|y-x|^{\alpha}}+\inf _{y \in \mathbb{R}^{n}, y \neq x} \frac{u(y)-u(x)}{|y-x|^{\alpha}},
$$

and $g$ grows at most like $|x|^{\beta}$ with $\beta<\alpha$ at infinity. For this problem, the corresponding of Theorem 1.5 and Theorem 1.8 will also hold true with $\partial \Omega$ replaced by $\mathbb{R}^{n} \backslash \Omega$. The crucial result is Lemma 8.3, which allows us to compare with functions of the type $|x|^{\beta}$.

\subsection{More general moduli of continuity}

Many of the results in this paper can be generalized when we replace $|x-y|^{\alpha}$ with some other modulus of continuity.

Consider a function $\omega: \mathbb{R}^{N} \rightarrow[0, \infty)$ such that

$$
\left\{\begin{array}{l}
\omega(x)>0=\omega(0) \quad \text { for all } \quad x \in \mathbb{R}^{N} \backslash\{0\} \\
\omega(x+y) \leq \omega(x)+\omega(y) \quad \text { for all } \quad x, y \in \mathbb{R}^{N}
\end{array}\right.
$$

Define for $x \in \Omega$

$$
\left(L_{\omega} u\right)(x)=\sup _{y \in \bar{\Omega}, y \neq x} \frac{u(y)-u(x)}{\omega(y-x)}+\inf _{y \in \bar{\Omega}, y \neq x} \frac{u(y)-u(x)}{\omega(y-x)} .
$$

For this case, in [16] a representation formula (Lem. 5.1) is found when $f=0$, and also the analogue of (iv) in Theorem 1.8 for the solution given by the representation formula, with the $C^{0, \alpha}$-regularity replaced by $C^{\omega}$-regularity.

It seems plausible that one can, following the ideas of the present paper, extend the following results to hold for the operators $L_{\omega}$ :

- The existence via Perron's method (Thm. 9.1), when $f$ has compact support.

- The comparison (Prop. 11.2), again under the assumption in Lemma 5.1.

\section{Open questions}

Some questions that remain unanswered in this paper that could be interesting to study in the future are listed below.

- The uniqueness for general functions $f$.

- Is the $C^{0, \alpha}$-regularity valid for general $f$, disprove or prove?

- What happens if we instead consider higher order operators of the form

$$
L u(x)=\sup _{\bar{\Omega} \backslash\{x\}} \frac{u(y)-u(x)-\nabla u(x) \cdot(x-y)}{|x-y|^{1+\alpha}}+\inf _{\bar{\Omega} \backslash\{x\}} \frac{u(y)-u(x)-\nabla u(x) \cdot(x-y)}{|x-y|^{1+\alpha}},
$$

with $\alpha \in[0,1]$. Will this yield in $C^{1, \alpha}$-extensions?

Acknowledgements. Along the development of this paper, all three authors have partially been supported by the ANR project "MICA", grant ANR-08-BLAN-0082. In addition, Erik Lindgren has been partially supported by the Chair "Mathematical modelling and numerical simulation, F-EADS - École Polytechnique - INRIA". The authors are also very grateful to Marco Cannone and Grzegorz Karch for providing very useful references.

\section{REFERENCES}

[1] G. Aronsson, Extension of functions satisfying Lipschitz conditions. Ark. Mat. 6 (1967) 551-561.

[2] G. Aronsson, On certain singular solutions of the partial differential equation $u_{x}^{2} u_{x x}+2 u_{x} u_{y} u_{x y}+u_{y}^{2} u_{y y}=0$. Manuscr. Math. 47 (1984) 133-151. 
[3] G. Aronsson, M.G. Crandall and P. Juutinen, A tour of the theory of absolutely minimizing functions. Bull. Am. Math. Soc. (N.S.) 41 (2004) 439-505.

[4] G. Barles, E. Chasseigne and C. Imbert, On the Dirichlet problem for second-order elliptic integro-differential equations. Indiana Univ. Math. J. 57 (2008) 213-246.

[5] T. Bhattacharya, E. Di Benedetto and J. Manfredi, Limits as $p \rightarrow \infty$ of $\Delta_{p} u_{p}=f$ and related extremal problems. Rend. Sem. Mat. Univ. Politec. Torino (1989), No. Special Issue (1991) 15-68, Some topics in nonlinear PDEs, Turin (1989).

[6] C. Bjorland, L. Caffarelli and A. Figalli, Non-Local Tug-of-War and the Infinity Fractional Laplacian. Comm. Pure Appl. Math. (2011).

[7] L.A. Caffarelli and A. Córdoba, An elementary regularity theory of minimal surfaces. Differential Integral Equations 6 (1993) $1-13$.

[8] V. Caselles, J.-M. Morel and C. Sbert, An axiomatic approach to image interpolation. IEEE Trans. Image Process. 7 (1998) $376-386$.

[9] M.G. Crandall, H. Ishii and P.-L. Lions, User's guide to viscosity solutions of second order partial differential equations. Bull. Amer. Math. Soc. (N.S.) 27 (1992) 1-67.

[10] E. Di Nezza, G. Palatucci and E. Valdinoci, Hitchhiker's guide to the fractional Sobolev spaces. preprint arXiv:1104.4345 (2011)

[11] H. Lebesgue, Sur le problème de Dirichlet. Rend. Circ. Mat. Palermo 24 (1907) 371-402.

[12] G. Lu and P. Wang, Inhomogeneous infinity Laplace equation. Adv. Math. 217 (2008) 1838-1868.

[13] E.J. McShane, Extension of range of functions. Bull. Am. Math. Soc. 40 (1934) 837-842.

[14] F. Memoli, J.-M. Sapiro and P. Thompson, Brain and surface warping via minimizing lipschitz extensions. IMA Preprint Series 2092 (2006).

[15] K. Murota, Discrete convex analysis. SIAM Monographs on Discrete Mathematics and Applications, Society for Industrial and Applied Mathematics (SIAM), Philadelphia, PA (2003).

[16] A.M. Oberman, An explicit solution of the Lipschitz extension problem. Proc. Am. Math. Soc. 136 (2008) 4329-4338.

[17] H. Whitney, Analytic extensions of differentiable functions defined in closed sets. Trans. Am. Math. Soc. 36 (1934) 63-89. 